\title{
An Extensive Operational Law for Monotone Functions of LR Fuzzy Intervals with Applications to Fuzzy Optimization
}

\section{Mingxuan Zhao}

Tongji University

Yulin Han

KU Leuven

Jian Zhou ( $\nabla$ zhou_jian@shu.edu.cn )

Shanghai University

\section{Research Article}

Keywords: Fuzzy interval , Monotone function , Operational law , Expected value , Fuzzy programming

Posted Date: May 25th, 2021

DOl: https://doi.org/10.21203/rs.3.rs-417449/v1

License: (c) This work is licensed under a Creative Commons Attribution 4.0 International License.

Read Full License 


\title{
An Extensive Operational Law for Monotone Functions of LR Fuzzy Intervals with Applications to Fuzzy Optimization
}

\author{
Mingxuan Zhao • Yulin Han • Jian Zhou
}

Received: date / Accepted: date

\begin{abstract}
The operational law put forward by Zhou et al. on strictly monotone functions with regard to regular LR fuzzy numbers makes a valuable push to the development of fuzzy set theory. However, its applicable conditions are confined to strictly monotone functions and regular LR fuzzy numbers, which restricts its application in practice to a certain degree. In this paper, we propose an extensive operational law that generalizes the one proposed by Zhou et al. to apply to monotone (but not necessarily strictly monotone) functions with regard to regular LR fuzzy intervals (LR-FIs), of which regular fuzzy numbers can be regarded as particular cases. By means of the extensive operational law, the inverse credibility distributions (ICDs) of monotone functions regarding regular LRFIs can be calculated efficiently and effectively. Moreover, the extensive operational law has a wider range of applications, which can deal with the situations hard to be handled by the original operational law. Subsequently, based on the extensive operational law, the computational formulae for expected values (EVs) of LR-FIs and monotone functions with regard to regular LR-FIs are presented. Furthermore, the
\end{abstract}

\footnotetext{
M. Zhao

School of Economics and Management, Tongji University, 200092, Shanghai, China

Y. Han

Faculty of Economics and Business, KU Leuven, 3000, Leuven, Belgium

J. Zhou $\bowtie$

School of Management, Shanghai University, Shanghai 200444, China

E-mail: zhou_jian@shu.edu.cn (J. Zhou )
}

proposed operational law is also applied to dispose fuzzy optimization problems with regular LR-FIs, for which a solution strategy is provided, where the fuzzy programming is converted to a deterministic equivalent first and then a newly-devised solution algorithm is utilized. Finally, the proposed solution strategy is applied to a purchasing planning problem, whose performances are evaluated by comparing with the traditional fuzzy simulation-based genetic algorithm. Experimental results indicate that our method is much more efficient, yielding highquality solutions within a short time.

Keywords Fuzzy interval · Monotone function · Operational law · Expected value · Fuzzy programming

\section{Introduction}

In real-life cases uncertainty on input parameters involved in some optimization problem$\mathrm{s}$ is inevitable due to the unpredictable natural factors. In this regard, fuzzy set theory initiated by Zadeh (1965) as one of the popular ways coping with uncertainties is applied to practical optimization process of various research fields, such as management (Ke et al. 2018), transportation (Büyüközkan et al. 2018), finance (Muzzioli and De Baets 2017) among others. Under the fuzzy circumstance, uncertain parameters are commonly assigned to be fuzzy variables, in which fuzzy numbers and fuzzy intervals (Dubois and Prade 1988) (or flat fuzzy numbers (Dubois and Prade 1979)) are two frequently used types. The main difference 
between them is that the modal value of fuzzy number is a point value, while the set of modal values of fuzzy interval is an interval. From the mathematical viewpoint, we can consider fuzzy numbers as a particular situation of fuzzy intervals. Therefore, the emphasis of research in this paper is placed on fuzzy intervals.

With regard to the parametric representation of fuzzy interval, Dubois and Prade (1979, 1988) defined a well-known L-R representation, in which $\mathrm{L}$ and $\mathrm{R}$ are shape functions representing the left and right sides of membership function respectively. From another point of view, Goetschel and Voxman (1986) proposed an equivalent representation named as L-U representation in accordance with the lower and upper branches, which define the two endpoints of an $\alpha$-cut. Stefanini and Guerra (2017) suggested ACF-representation to describe a fuzzy interval by using a new defined average cumulative function on the basis of the possibility theory. It should be mentioned that the typical L-R representation in Dubois and Prade (1988) is utilized to delineate fuzzy interval in this paper, and the corresponding fuzzy interval is termed as LR-FI accordingly.

As an effective and efficient tool used in fuzzy optimization, fuzzy arithmetic has attracted interests of many researchers. Zadeh (1975) initially extended the common arithmetic operations for real numbers to fuzzy intervals via the proposed extension principle on the basis of a triangular norm (t-norm). As for the t-normbased arithmetic operations, an important feature is to offer a way for controlling the rise on uncertainty in the process of computations, and avoid variables shifting off their most vital values simultaneously. However, the practical use of Zadeh's extension principle is a little complicated owing to the involved nonlinear operators. Subsequently, Dubois and Prade (1979) proposed some analytical calculations including the basic arithmetic addition, subtraction, multiplication, and division among LR-FIs together with some properties. Meanwhile, there is some other literature focusing on algebraic operations of fuzzy intervals. Hwang and Lee (2001) studied the sum for LR-FIs in accordance with a given nilpotent t-norm, which modified the form of output sum in Markova (1997) to the one with arbitrary spreads, and generalized the results of Hong and Hwang (1997) for the membership function of the sum for LR-
FIs. For the sake of preserving the shapes of fuzzy intervals during the practical computation, some shape-preserving operations on fuzzy intervals with sigmoid and bell-shaped membership functions (Dombi and Gyorbiro 2006; Hong 2007; Hong et al. 2007) were investigated. With the same aim, Mak (2012) constructed the real vector space of LR-FIs, and then presented the algebraic forms and the associated application. Based upon the definition of unrestricted LRFI (Kaur and Kumar 2012), Kaur and Kumar (2013) presented the product of unconstrained LR-FI, thereby formulating a Mehar's method to deal with the fully linear programming problems. Additionally, some operations on a specific type of fuzzy intervals like trapezoidal fuzzy numbers are discussed by Abbasi and Allahviranloo (2019), Shakeel et al. (2019a,b), etc.

As a particular kind of LR-FIs, LR fuzzy numbers have got quite a few attentions because of the good interpretability and easy performing for usual operations since they were introduced by Dubois and Prade Dubois and Prade (1978). So far there has been many relevant studies on arithmetic operations of LR fuzzy numbers (see, e.g., Ban et al. 2016; Chou 2003; Oussalah and De Schutter 2003; Zhou et al. 2016). In particular, on account of the credibility measure pioneered by Liu (2002), Zhou et al. (2016) proposed an operational law targeting at strictly monotone functions with regard to LR fuzzy numbers. Based on this, a crispy solution framework for the fuzzy programming was formulated, which reduces the computation complexity a lot. Given the effectiveness of the operational law in Zhou et al. (2016), it has been gradually employed to dispose of sorts of optimization problems. For example, Zhong et al. (2019) devised a two-phase approach by integrating the operational law in Zhou et al. (2016) into a genetic algorithm to handle with the preventive maintenance scheduling problem. Yang et al. (2019) developed an improved method to tackle a fuzzy facility location problem by using the operational law proposed in Zhou et al. (2016). Besides, the research findings in Zhou et al. (2016) are also applied to other fields such as location problem (Soltanpour et al. 2019), quality function deployment (Liu et al. 2016), evaluation for the air quality (Wang et al. 2017), green-fuzzy vehicle routing problem (Wang et al. 2018), diagnosis of prostate cancer (Kar and Majumder 2017), and so on. 
Although the operational law in Zhou et al. (2016) is quite practical and applicable to some fuzzy optimization problems, it is notable that the functions in the fuzzy programming models are restricted to strictly monotone ones in regard to fuzzy variables and the involving fuzzy variables are required to be regular LR fuzzy numbers such as triangular fuzzy numbers. In practice, many optimization problems cannot be modeled by strictly monotone functions like classical newsvendor problem and moreover as for the fuzzy variables there are too many cases where the operational law in Zhou et al. (2016) cannot work, for example, when those fuzzy variables are regular LR-FIs defined by Liu et al. (2020) like trapezoidal fuzzy numbers. Therefore, it is necessary and valuable to make an extensive study for the research of Zhou et al. (2016).

The major contributions of this paper to the field of fuzzy arithmetics and fuzzy optimization are fourfold.

1. We propose the ICD of an LR-FI based on credibility measure, which is a generalization for the ICD of regular LR fuzzy number defined in Zhou et al. (2016), and verify two equivalent conditions of regular LR-FI.

2. We present an extensive operational law on monotone functions with regard to regular LR-FIs, which generalizes the operational law in Zhou et al. (2016) from both the function monotonicity and the type of fuzzy variables. Concretely, the strictly monotone functions are extended to be monotone (but not necessarily strictly monotone) function$\mathrm{s}$, and the regular LR fuzzy numbers are generalized to regular LR-FIs such as trapezoidal fuzzy numbers.

3. We develop calculation formulas for EVs of LR-FIs and monotone functions with regard to regular LR-FIs on the basis of the extensive operational law. In accordance with the calculation formulas, the EVs of monotone functions of regular LR-FIs can be directly derived by means of the corresponding ICDs.

4. We construct a solution strategy including a newly-devised heuristic algorithm with a new effective fuzzy simulation for the fuzzy chance-constrained programming (CCP) with monotone functions of regular LR-FIs. Then we apply it to solve a fuzzy purchasing planning problem. Based on the numerical ex- periments, our method shows better performances on both solution accuracy and efficiency in comparison with another traditional heuristic algorithm.

The remaining of this paper is organized as follows. Section 2 recalls some fundamental notions regarding the LR-FI, defines its ICD in the light of the credibility distribution, and then derives the equivalent conditions of regular LRFIs. In Section 3, we explore the property of monotone functions with regard to regular LRFIs, propose a new operational law and then discuss the EVs of LR-FIs and monotone functions in regard to regular LR-FIs. In Section 4, a solution strategy for the fuzzy CCP is formulated based on the new operational law. To exhibit the effectiveness of our strategy, some numerical experiments are implemented by using our method and a traditional heuristic method respectively in the context of a purchasing planning problem. Finally, the main findings are concluded in Section 5. The conceptual framework of our study is demonstrated in Figure 1.

\section{LR fuzzy interval and its inverse credibility distribution}

In this section, some elementary conceptions in relation to LR-FI and the credibility distribution of fuzzy variable are reviewed first. We subsequently define the ICD of an LR-FI, and derive its mathematical expression. After that we introduce the definition of the regular LR-FI and prove its two necessary and sufficient conditions.

\subsection{LR fuzzy interval and its credibility} distribution

The well-known LR-FI was initially proposed by Dubois and Prade (1988), in which $L$ and $R$ are the decreasing left and right shape functions from $[0, \infty) \rightarrow[0,1]$ with $L(0)=1$ and $R(0)=$ 1 respectively.

Definition 1 (Dubois and Prade 1988) A fuzzy interval $\widetilde{M}$ defined on universal set of real numbers $\mathbb{R}$ is said to be an LR-FI if it has the membership function with shape functions $L, R$ and 


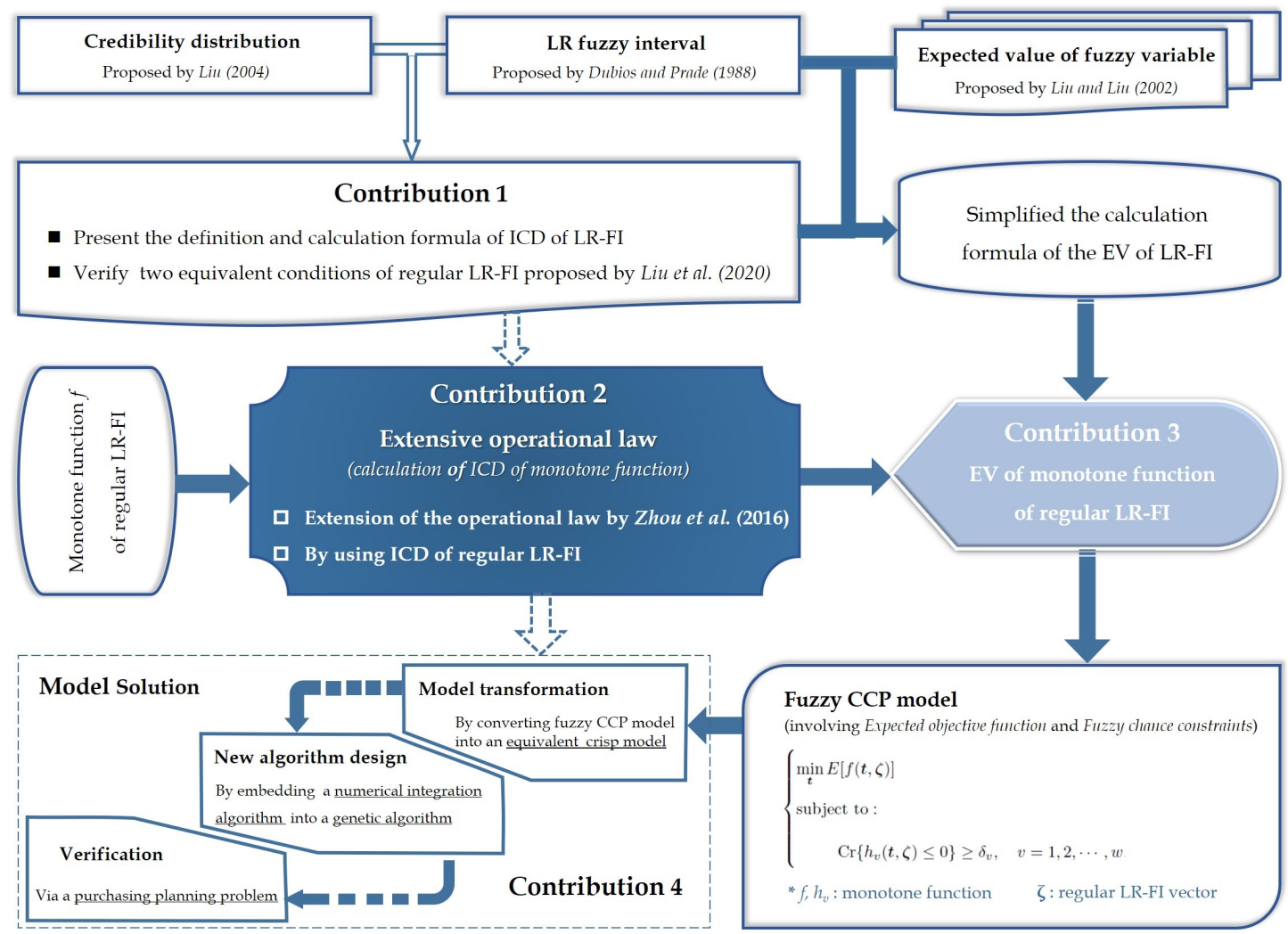

Fig. 1 Conceptual framework of our study

four parameters $\underline{c}, \bar{c}, \rho>0, \sigma>0$ as

$\nu(t)= \begin{cases}L\left(\frac{\underline{c}-t}{\rho}\right), & \text { if } t<\underline{c} \\ 1, & \text { if } \underline{c} \leq t \leq \bar{c} \\ R\left(\frac{t-\bar{c}}{\sigma}\right), & \text { if } t>\bar{c},\end{cases}$

where $[\underline{c}, \bar{c}]$ is the core of $\widetilde{M}, \underline{c}$ and $\bar{c}$ are respectively called the lower and upper modal values, $\rho$ and $\sigma$ are respectively called the left and right spreads. More briefly, the fuzzy interval $\widetilde{M}$ is denoted by $(\underline{c}, \bar{c}, \rho, \sigma)_{L R}$.

Remark 1: If $\rho$ and $\sigma$ are both equal to 0 , that is, $\widetilde{M}=(\underline{c}, \bar{c}, 0,0)_{L R}$ for all $L$ and $R$, then $\widetilde{M}$ degenerates into a real interval as $[\underline{c}, \bar{c}]$. If the core of $\widetilde{M}$ is a real number $\underline{c}$ (i.e., $\underline{c}=\bar{c}$ ), then $\widetilde{M}$ degrades into an LR fuzzy number denoted by $(\underline{c}, \rho, \sigma)_{L R}$. Furthermore, when $\widetilde{M}=$ $(\underline{c}, \underline{c}, 0,0)_{L R}$ for all $L$ and $R, \widetilde{M}$ is just a real number $\underline{c} \in \mathbb{R}$.

For the subsequent research on the properties of fuzzy events in the fuzzy world, the credibility measure proposed by Liu and Liu (2002) is introduced here to measure the credibility of a fuzzy event. If $t$ is a real number and $\zeta$ is a fuzzy variable with membership function $\nu$, then the credibility of fuzzy event $\{\zeta \leq t\}$ can be calculated by

$\operatorname{Cr}\{\zeta \leq t\}=\frac{1}{2}\left(\sup _{y \leq t} \nu(y)+1-\sup _{y>t} \nu(y)\right)$

To describe fuzzy variables, credibility distributions as a carrier of incomplete information of those variables were defined by Liu (2004) as follows.

Definition 2 (Liu 2004) If $\zeta$ is a fuzzy variable, then its credibility distribution $\psi: \mathbb{R} \rightarrow$ $[0,1]$ is defined by

$\psi(t)=\operatorname{Cr}\{\zeta \leq t\}$

In accordance with the mathematical properties of credibility measure, it is known that the credibility distribution $\psi$ is non-decreasing on $\mathbb{R}$, in which $\psi(-\infty)=0$ and $\psi(+\infty)=1$.

As for the LR-FI $\widetilde{M}=(\underline{c}, \bar{c}, \rho, \sigma)_{L R}$ with the membership function $\nu$ in Eq. (1), on account of Eqs. (2)-(3), its credibility distribution can 
be derived as

$$
\psi(t)= \begin{cases}0.5 L\left(\frac{\underline{c}-t}{\rho}\right), & \text { if } t<\underline{c} \\ 0.5, & \text { if } \underline{c} \leq t \leq \bar{c} \\ 1-0.5 R\left(\frac{t-\bar{c}}{\sigma}\right), & \text { if } t>\bar{c} .\end{cases}
$$

Example 1 If an LR-FI $(\underline{c}, \bar{c}, \rho, \sigma)_{L R}$ has the shape functions $L(t)=R(t)=\max \{0,1-t\}$, then it is called trapezoidal fuzzy number denoted by $\mathcal{T}(\underline{c}, \bar{c}, \rho, \sigma)_{L R}$ with the membership function and credibility distribution

$\nu(t)= \begin{cases}\frac{t+\rho-\underline{c}}{\rho}, & \text { if } \underline{c}-\rho \leq t<\underline{c} \\ 1, & \text { if } \underline{c} \leq t \leq \bar{c} \\ \frac{\bar{c}+\sigma-t}{\sigma}, & \text { if } \bar{c}<t \leq \bar{c}+\sigma \\ 0, & \text { otherwise }\end{cases}$

and

$$
\psi(t)= \begin{cases}0, & \text { if } t<\underline{c}-\rho \\ \frac{t+\rho-\underline{c}}{2 \rho}, & \text { if } \underline{c}-\rho \leq t<\underline{c} \\ 0.5, & \text { if } \underline{c} \leq t \leq \bar{c} \\ \frac{t+\sigma-\bar{c}}{2 \sigma}, & \text { if } \bar{c}<t \leq \bar{c}+\sigma \\ 1, & \text { if } t>\bar{c}+\sigma,\end{cases}
$$

as depicted in Figures 2(a) and 2(b), respectively.

Example 2 If an LR-FI $(\underline{c}, \bar{c}, \rho, \sigma)_{L R}$ has the shape functions $L(t)=R(t)=\max \left\{0,1-t^{2}\right\}$, denoted by $\mathcal{A}(\underline{c}, \bar{c}, \rho, \sigma)_{L R}$, then it has the membership function

$\nu(t)= \begin{cases}1-\frac{(\underline{c}-t)^{2}}{\rho^{2}}, & \text { if } \underline{c}-\rho \leq t<\underline{c} \\ 1, & \text { if } \underline{c} \leq t \leq \bar{c} \\ 1-\frac{(t-\bar{c})^{2}}{\sigma^{2}}, & \text { if } \bar{c}<t \leq \bar{c}+\sigma \\ 0, & \text { otherwise }\end{cases}$

and the credibility distribution

$$
\psi(t)= \begin{cases}0, & \text { if } t<\underline{c}-\rho \\ 0.5-\frac{(\underline{c}-t)^{2}}{2 \rho^{2}}, & \text { if } \underline{c}-\rho \leq t<\underline{c} \\ 0.5, & \text { if } \underline{c} \leq t \leq \bar{c} \\ 0.5+\frac{(t-\bar{c})^{2}}{2 \sigma^{2}}, & \text { if } \bar{c}<t \leq \bar{c}+\sigma \\ 1, & \text { if } t>\bar{c}+\sigma,\end{cases}
$$

as depicted in Figures 3(a) and 3(b), respectively.

Example 3 If an LR-FI $(\underline{c}, \bar{c}, \rho, \sigma)_{L R}$ has the shape functions $L(t)=\max \{1-t, 0\}$ and $R(t)=$ $\max \left\{1-t^{2}, 0\right\}$, denoted by $\mathcal{B}(\underline{c}, \bar{c}, \rho, \sigma)_{L R}$, then it has the membership function

$\nu(t)= \begin{cases}\frac{t+\rho-\underline{c}}{\rho}, & \text { if } \underline{c}-\rho \leq t<\underline{c} \\ 1, & \text { if } \underline{c} \leq t \leq \bar{c} \\ 1-\frac{(t-\bar{c})^{2}}{\sigma^{2}}, & \text { if } \bar{c}<t \leq \bar{c}+\sigma \\ 0, & \text { otherwise }\end{cases}$

and the credibility distribution

$\psi(t)= \begin{cases}0, & \text { if } t<\underline{c}-\rho \\ \frac{t+\rho-\underline{c}}{2 \rho}, & \text { if } \underline{c}-\rho \leq t<\underline{c} \\ 0.5, & \text { if } \underline{c} \leq t \leq \bar{c} \\ 0.5+\frac{(t-\bar{c})^{2}}{2 \sigma^{2}}, & \text { if } \bar{c}<t \leq \bar{c}+\sigma \\ 1, & \text { if } t>\bar{c}+\sigma,\end{cases}$

as depicted in Figures 4(a) and 4(b), respectively.

Example 4 If an LR-FI $(2,4,2,4)_{L R}$ has the following shape functions

$L(t)= \begin{cases}1, & \text { if } t=0 \\ 0.6, & \text { if } 0<t \leq 0.5 \\ 0.3, & \text { if } 0.5<t \leq 1 \\ 0, & \text { otherwise }\end{cases}$

and

$R(t)= \begin{cases}-0.8 t+1, & \text { if } 0 \leq t \leq 0.25 \\ 0.8, & \text { if } 0.25<t \leq 0.5 \\ -1.6 t+1.6, & \text { if } 0.5<t \leq 1 \\ 0, & \text { otherwise, }\end{cases}$

then it can be deduced that it has the membership function and credibility distribution

$\nu(t)= \begin{cases}0.3, & \text { if } 0 \leq t<1 \\ 0.6, & \text { if } 1 \leq t<2 \\ 1, & \text { if } 2 \leq t \leq 4 \\ -0.2 t+1.8, & \text { if } 4<t \leq 5 \\ 0.8, & \text { if } 5<t \leq 6 \\ -0.4 t+3.2, & \text { if } 6 \leq t \leq 8 \\ 0, & \text { otherwise }\end{cases}$ 


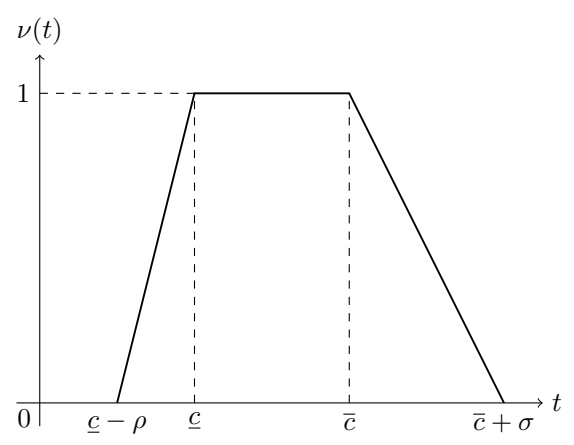

(a) The membership function

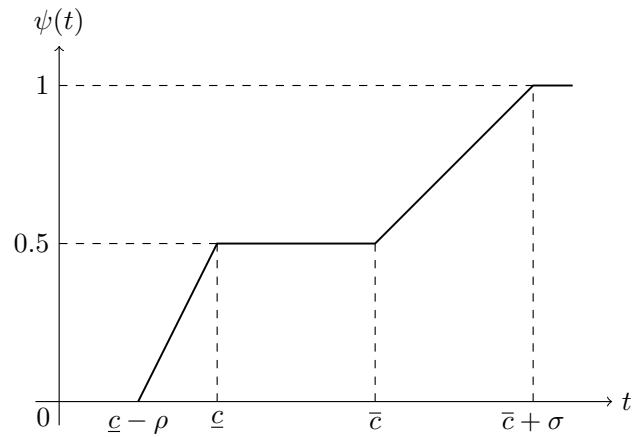

(b) The credibility distribution

Fig. 2 The membership function and credibility distribution of $\mathcal{T}(\underline{c}, \bar{c}, \rho, \sigma)_{L R}$ in Example 1

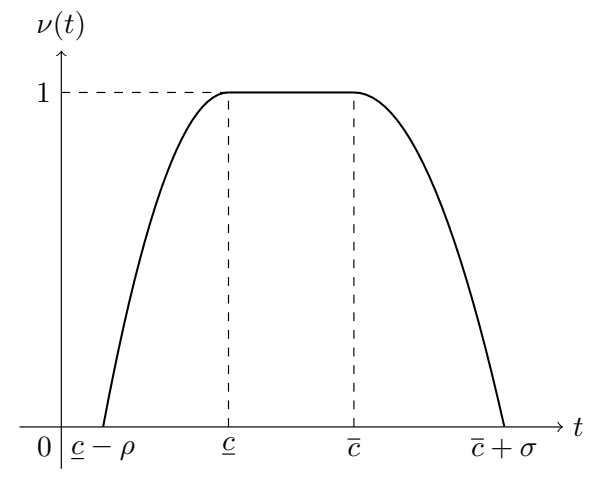

(a) The membership function

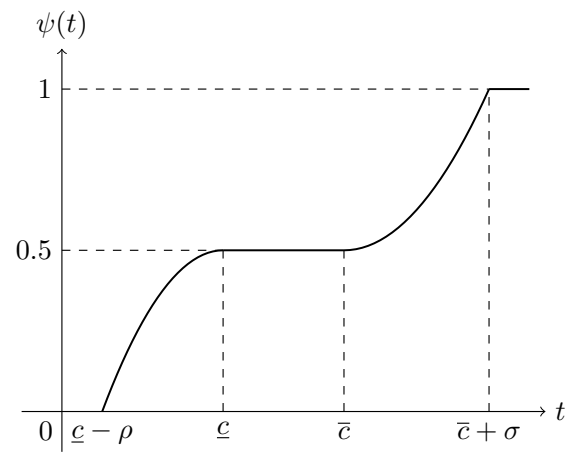

(b) The credibility distribution

Fig. 3 The membership function and credibility distribution of $\mathcal{A}(\underline{c}, \bar{c}, \rho, \sigma)_{L R}$ in Example 2

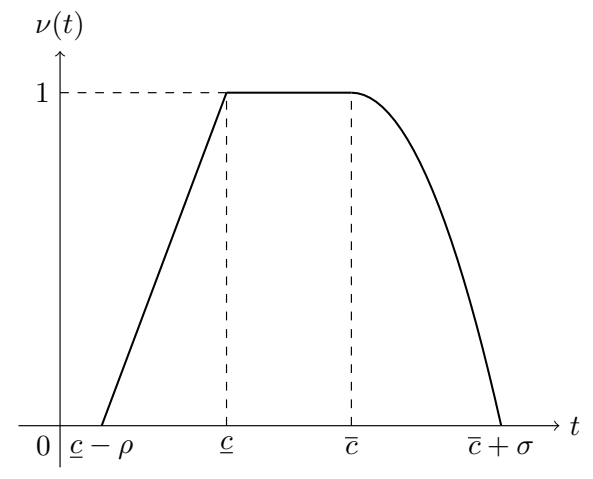

(a) The membership function

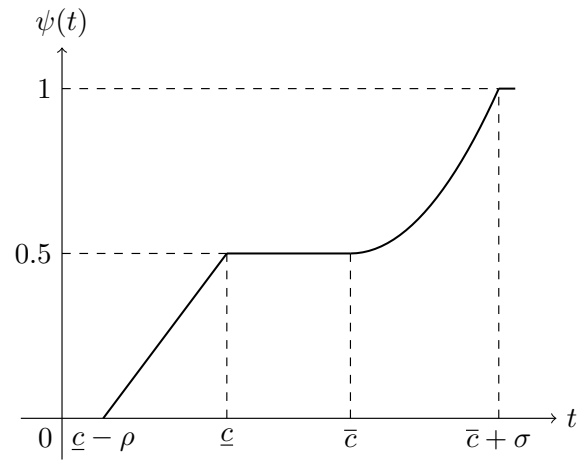

(b) The credibility distribution

Fig. 4 The membership function and credibility distribution of $\mathcal{B}(\underline{c}, \bar{c}, \rho, \sigma)_{L R}$ in Example 3 
and

$$
\psi(t)= \begin{cases}0, & \text { if } t \leq 0 \\ 0.15, & \text { if } 0<t<1 \\ 0.3, & \text { if } 1 \leq t<2 \\ 0.5, & \text { if } 2 \leq t<4 \\ 0.1 t+0.1, & \text { if } 4 \leq t<5 \\ 0.6, & \text { if } 5 \leq t<6 \\ 0.2 t-0.6, & \text { if } 6 \leq t<8 \\ 1, & \text { if } t \geq 8,\end{cases}
$$

as depicted in Figures 5(a) and 5(b), respectively.

2.2 Inverse credibility distribution of LR fuzzy interval

For our purpose, the ICD of an LR-FI is defined as below, which will play an important role then.

Definition 3 Let $\zeta$ be an LR-FI. A multi-valued function $F:[0,1] \mapsto \mathbb{R}$ is called the ICD of $\zeta$ if

$\operatorname{Cr}\left\{\zeta \leq f_{\delta}\right\}=\bar{\delta}, \quad \delta \in[0,1]$,

where $f_{\delta} \in\{t \mid t=F(\delta)\}$ and $\bar{\delta}=\sup \{\gamma \mid F(\gamma)=$ $F(\delta)\}$.

Remark 2: For simplicity, $F(\delta)$ is denoted by $\psi^{-1}(\delta)$, which differs from the inverse function of $\psi(t)$.

Theorem 1 Let $\psi(t)$ be the credibility distribution of an LR-FI $\zeta$, and $D_{\psi}$ be the domain of values of $\psi(t)$. Then the ICD of $\zeta$ is deduced as

$$
\psi^{-1}(\delta)= \begin{cases}\sup \{t \mid \psi(t)=0\}, & \text { if } \delta=0 \\ \{t \mid \psi(t)=\delta\}, & \text { if } 0<\delta<1 \\ & \text { and } \delta \in D_{\psi} \\ \inf \{t \mid \psi(t) \geq \delta\}, & \text { if } 0<\delta<1 \\ & \text { and } \delta \notin D_{\psi} \\ \inf \{t \mid \psi(t)=1\}, & \text { if } \delta=1 .\end{cases}
$$

Proof: The proof is provided in Appendix B.
Example 5 The ICD of $\mathcal{T}(\underline{c}, \bar{c}, \rho, \sigma)_{L R}$ in Example 1 is (see Figure 6)

$\psi^{-1}(\delta)= \begin{cases}2 \rho \delta+\underline{c}-\rho, & \text { if } 0 \leq \delta<0.5 \\ {[\underline{c}, \bar{c}],} & \text { if } \delta=0.5 \\ 2 \sigma \alpha+\bar{c}-\sigma, & \text { if } 0.5<\delta \leq 1 .\end{cases}$

Example 6 The ICD of $\mathcal{A}(\underline{c}, \bar{c}, \rho, \sigma)_{L R}$ in Example 2 is (see Figure 7 )

$\psi^{-1}(\delta)= \begin{cases}\underline{c}-\rho \sqrt{1-2 \delta}, & \text { if } 0 \leq \delta<0.5 \\ {[\underline{c}, \bar{c}],} & \text { if } \delta=0.5 \\ \bar{c}+\sigma \sqrt{2 \delta-1}, & \text { if } 0.5<\delta \leq 1 .\end{cases}$

Example 7 The ICD of $\mathcal{B}(\underline{c}, \bar{c}, \rho, \sigma)_{L R}$ in Example 3 is (see Figure 8 )

$\psi^{-1}(\delta)= \begin{cases}2 \rho \delta+\underline{c}-\rho, & \text { if } 0 \leq \delta<0.5 \\ {[\underline{c}, \bar{c}],} & \text { if } \delta=0.5 \\ \bar{c}+\sigma \sqrt{2 \delta-1}, & \text { if } 0.5<\delta \leq 1 .\end{cases}$

Example 8 The ICD of the LR-FI $(2,4,2,4)_{L R}$ in Example 4 is (see Figure 9)

$\psi^{-1}(\delta)= \begin{cases}(-\infty, 0], & \text { if } \delta=0 \\ 0, & \text { if } 0<\delta<0.15 \\ (0,1), & \text { if } \delta=0.15 \\ 1, & \text { if } 0.15<\delta<0.3 \\ {[1,2),} & \text { if } \delta=0.3 \\ 2, & \text { if } 0.3<\delta<0.5 \\ {[2,4),} & \text { if } \delta=0.5 \\ 10 \delta-1, & \text { if } 0.5 \leq \delta<0.6 \\ {[5,6),} & \text { if } \delta=0.6 \\ 5 \delta+3, & \text { if } 0.6 \leq \delta<1 \\ {[8,+\infty),} & \text { if } \delta=1\end{cases}$

\subsection{Regular LR fuzzy interval}

It is worth noting that that the credibility distributions $\psi(t)$ (or the ICDs $\psi^{-1}(\delta)$ ) of LR-FIs in Examples 1-3 (or in Examples 5-7) are continuous and strictly increasing on the domain $\{t \mid 0<\psi(t)<0.5$ or $0.5<\psi(t)<1\}$. For the sake of describing such kind of LR-FIs, we first introduce the definition of regular LR-FI proposed in Liu et al. (2020) and then verify two equivalent conditions.

Definition 4 (Liu et al. 2020) If the shape functions $L$ and $R$ of an LR-FI $\zeta$ are continuous and strictly decreasing on the domains $\{t \mid 0<$ $L(t)<1\}$ and $\{t \mid 0<R(t)<1\}$, respectively, then the LR-FI is regular. 


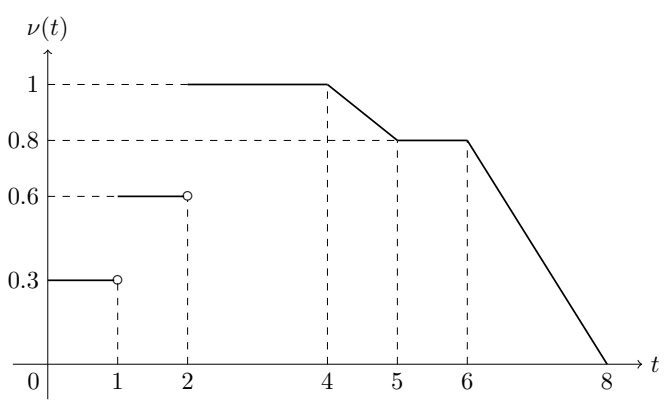

(a) The membership function

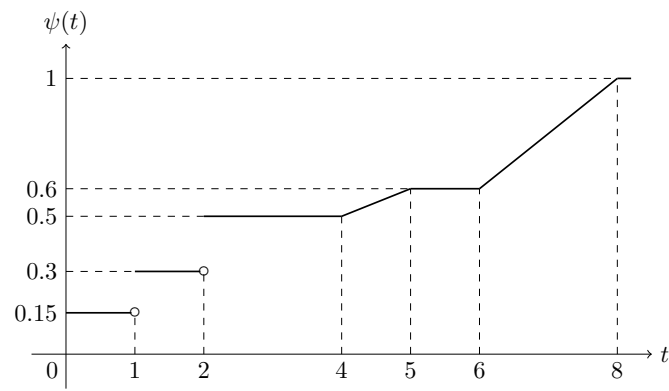

(b) The credibility distribution

Fig. 5 The membership function and credibility distribution of the LR-FI $(2,4,2,4)_{L R}$ in Example 4

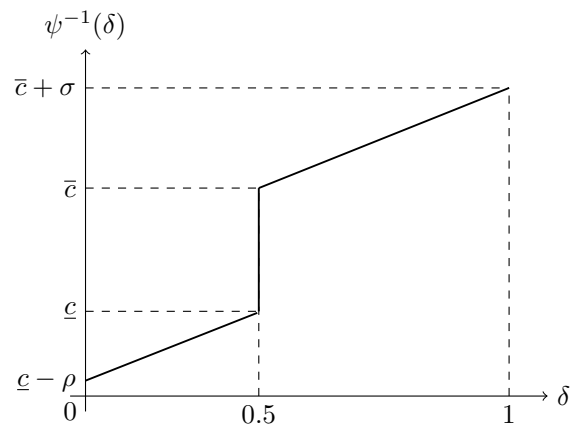

Fig. 6 The ICD of $\mathcal{T}(\underline{c}, \bar{c}, \rho, \sigma)_{L R}$ in Example 1

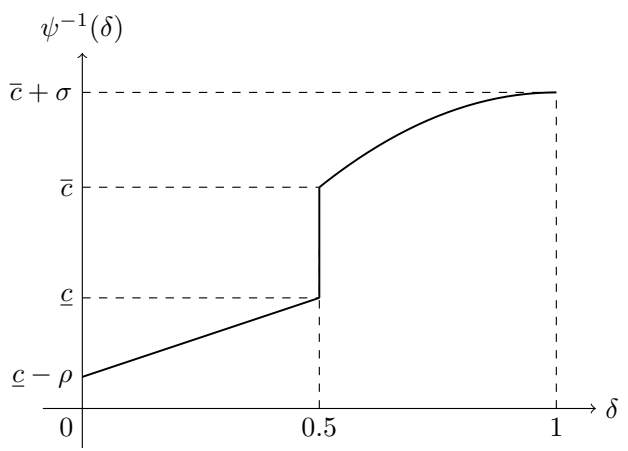

Fig. 8 The ICD of $\mathcal{B}(\underline{c}, \bar{c}, \rho, \sigma)_{L R}$ in Example 3

Theorem 2 An LR-FI is regular if and only if it satisfies any one of the following conditions, (i) The credibility distribution $\psi(t)$ is continuous on the domain $\{t \mid 0<\psi(t)<1\}$ and strictly increasing on the domain $\{t \mid 0<\psi(t)<$ 0.5 or $0.5<\psi(t)<1\}$;

(ii) The ICD $\psi^{-1}(\delta)$ is continuous on $(0,1)$ and strictly increasing on $(0,0.5) \cup(0.5,1)$.

Proof: This theorem follows from Eq. (4) and Definitions 3-4 immediately.

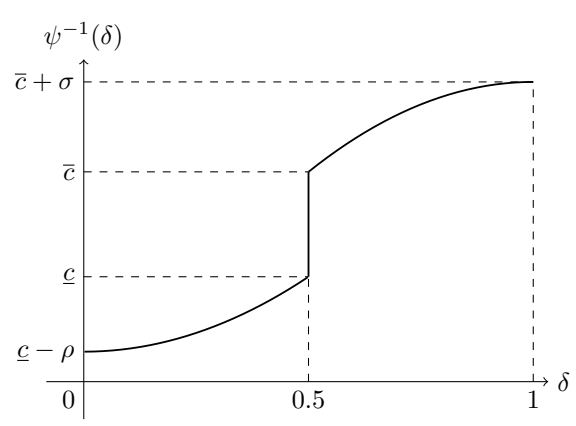

Fig. 7 The ICD of $\mathcal{A}(\underline{c}, \bar{c}, \rho, \sigma)_{L R}$ in Example 2

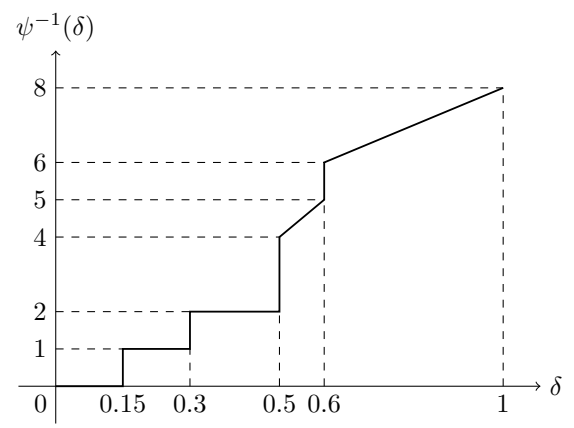

Fig. 9 The ICD of the LR-FI $(2,4,2,4)_{L R}$ in Example 4

As regards regular LR-FI, it seems clear that its $L$ and $R$ shape functions are both continuous and strictly increasing on respective domains, which also means that there exist the inverse functions of shape functions, i.e., $L^{-1}$ and $R^{-1}$. Thus it follows from the above analysis and the definition of regular LR-FI that the ICD of a regular LR-FI can be deduced directly. 
Theorem 3 Let $\zeta=(\underline{c}, \bar{c}, \rho, \sigma)_{L R}$ be a regular LR-FI. Then its ICD is derived as

$$
\psi^{-1}(\delta)= \begin{cases}\underline{c}-\rho L^{-1}(2 \delta), & \text { if } 0 \leq \delta<0.5 \\ {[\underline{c}, \bar{c}],} & \text { if } \delta=0.5 \\ \bar{c}+\sigma R^{-1}(2-2 \delta), & \text { if } 0.5<\delta \leq 1 .\end{cases}
$$

Proof: This theorem follows from Eq. (4) and Theorem 1.

\section{Operational law and expected value for monotone function of regular LR fuzzy intervals}

This section first recalls the the concept of monotonicity of functions, and discusses the property of credibility distributions of monotone functions with regard to regular LR-FIs. On this basis, we put forward a novel operational law on monotone function with regard to regular LR-FIs and further explore its EV.

3.1 Monotone function of regular LR fuzzy intervals

At present the monotonicity of a function is defined by many scholars from various perspectives. The definition of monotone function and strictly monotone function proposed by Liu et al. (2016) and Liu (2010) is employed in this paper.

Definition 5 (Liu et al. 2016 and Liu 2010) $A$ real-valued function $f\left(t_{1}, t_{2}, \cdots, t_{n}\right)$ is called monotone function if it is increasing regarding $t_{1}, t_{2}, \cdots, t_{k}$ and decreasing regarding $t_{k+1}, t_{k+2}$, $\cdots, t_{n}$, that is,

$$
f\left(t_{1}, \cdots, t_{k}, t_{k+1}, \cdots, t_{n}\right) \geq f\left(s_{1}, \cdots, s_{k}, s_{k+1},\right.
$$$$
\left.\cdots, s_{n}\right)
$$

holds for any $t_{i} \geq s_{i}$ for $i=1,2, \cdots, k$ and $t_{i} \leq s_{i}$ for $i=k+1, \cdots, n$. Moreover, if the function $f\left(t_{1}, t_{2}, \cdots, t_{n}\right)$ satisfies

$$
\begin{array}{r}
f\left(t_{1}, \cdots, t_{k}, t_{k+1}, \cdots, t_{n}\right)>f\left(s_{1}, \cdots, s_{k}, s_{k+1},\right. \\
\left.\cdots, s_{n}\right)
\end{array}
$$

for any $t_{i}>s_{i}$ for $i=1,2, \cdots, k$ and $t_{i}<s_{i}$ for $i=k+1, \cdots, n$, then it is said to be strictly monotone.

Remark 3: A real-valued function $f\left(t_{1}, t_{2}, \cdots\right.$, $t_{n}$ ) is called increasing (decreasing) function regarding $t_{1}, t_{2}, \cdots, t_{n}$ if $f\left(t_{1}, t_{2}, \cdots, t_{n}\right) \geq f\left(s_{1}\right.$, $\left.s_{2}, \cdots, s_{n}\right)$ holds for any $t_{i} \geq s_{i}\left(t_{i} \leq s_{i}\right)$ for $i=$ $1,2, \cdots, n$. Moreover, if the function $f\left(t_{1}, t_{2}, \cdots\right.$ ,$\left.t_{n}\right)$ satisfies $f\left(t_{1}, t_{2}, \cdots, t_{n}\right)>f\left(s_{1}, s_{2}, \cdots, s_{n}\right)$ for any $t_{i}>s_{i}\left(t_{i}<s_{i}\right)$ for $i=1,2, \cdots, n$, then it is called strictly increasing (decreasing) function.

Example 9 The following functions are strictly monotone,

$$
\begin{gathered}
f\left(t_{1}, t_{2}\right)=t_{1}-t_{2}, \\
f\left(t_{1}, t_{2}\right)=t_{1} \times t_{2}, \quad t_{1}, t_{2}>0 .
\end{gathered}
$$

Example 10 The following functions are monotone but not strictly monotone,

$$
f\left(t_{1}, t_{2}\right)=a \vee t_{1}-b \wedge t_{2},
$$

$$
f\left(t_{1}, t_{2}\right)=a \vee t_{1} /\left(b \wedge t_{2}\right), \quad a \vee t_{1}, b \wedge t_{2}>0 .
$$

In some practical optimization problems, the objective functions of the formulated optimization models are usually monotone but not strictly monotone, such as the well-known newsvendor problem, inventory problem, project scheduling problem, etc. Considering the generality of monotone functions in practical applications, we will proceed to analyse the property of continuous and monotone (but not necessarily strictly monotone) functions of regular LR-FIs.

Theorem 4 Let $\zeta_{1}, \zeta_{2}, \cdots, \zeta_{n}$ be independent regular LR-FIs and $f: \mathbb{R}^{n} \rightarrow \mathbb{R}$ a continuous and monotone function. Then $\zeta=f\left(\zeta_{1}, \zeta_{2}, \cdots\right.$, $\left.\zeta_{n}\right)$ is an $L R-F I$.

Proof: The proof is provided in Appendix C.

Example 11 Let $\zeta_{1} \sim \mathcal{T}(2,4,2,3)_{L R}$. The function $f_{1}$

$f_{1}(t)= \begin{cases}t-1, & \text { if } t<3 \\ 2, & \text { if } 3 \leq t \leq 5 \\ t-3, & \text { if } t>5\end{cases}$ 
is increasing but not strictly increasing. Then the credibility distribution of $f_{1}\left(\zeta_{1}\right)$ is obtained as follows

$$
\psi(t)= \begin{cases}0, & \text { if } t<-1 \\ \frac{t+1}{4}, & \text { if }-1 \leq t<1 \\ \frac{1}{2}, & \text { if } 1 \leq t \leq 2 \\ \frac{t+2}{6}, & \text { if } 2<t \leq 4 \\ 1, & \text { if } t>4,\end{cases}
$$

as depicted in Figure 10. It can be concluded from Figure 10 that $f_{1}\left(\zeta_{1}\right)$ is not a regular LRFI.

\subsection{Operational law}

On account of the extensive applications of monotone functions and regular LR-FIs in optimization problems, a new operational law is proposed in this subsection, which can be considered as an extension to the one developed in Zhou et al. (2016).

Theorem 5 Let $\zeta_{1}, \zeta_{2}, \cdots, \zeta_{n}$ be independen$t$ regular LR-FIs with ICD $\psi_{1}^{-1}, \psi_{2}^{-1}, \cdots, \psi_{n}^{-1}$, respectively. If the continuous function $f\left(t_{1}, t_{2}\right.$, $\left.\cdots, t_{n}\right)$ is increasing regarding $t_{1}, t_{2}, \cdots, t_{k}$ and decreasing regarding $t_{k+1}, t_{k+2}, \cdots, t_{n}$, then $\zeta=$ $f\left(\zeta_{1}, \zeta_{2}, \cdots, \zeta_{n}\right)$ is an LR-FI with the ICD

$$
\begin{aligned}
\psi^{-1}(\delta)= & f\left(\psi_{1}^{-1}(\delta), \cdots, \psi_{k}^{-1}(\delta), \psi_{k+1}^{-1}(1-\delta),\right. \\
& \left.\cdots, \psi_{n}^{-1}(1-\delta)\right) .
\end{aligned}
$$

Proof: The proof is provided in Appendix D.

Example 12 Let $\zeta_{1}$ be a trapezoidal fuzzy number denoted by $\mathcal{T}(2,4,2,3)_{L R}$ with the credibility distribution $\psi_{1}$. Then the ICD of $f_{1}\left(\zeta_{1}\right)$ with $f_{1}$ defined in Eq. (20) is deduced from Theorem 5 as

$$
\begin{aligned}
\psi_{f_{1}}^{-1}(\delta)= & f_{1}\left(\psi_{1}^{-1}(\delta)\right) \\
& =\left\{\begin{array}{lll}
4 \delta-1, & \text { if } & 0 \leq \delta<\frac{1}{2} \\
{[1,2],} & \text { if } \quad \delta=\frac{1}{2} \\
2, & \text { if } \quad \frac{1}{2}<\delta \leq \frac{2}{3} \\
6 \delta-2, & \text { if } \quad \frac{2}{3}<\delta \leq 1
\end{array}\right.
\end{aligned}
$$

as depicted in Figure 11.

Example 13 Let $\zeta_{2}$ be a trapezoidal fuzzy number denoted by $\mathcal{T}(1,2,2,1)_{L R}$ with the credibility distribution $\psi_{2}$. Then by using the operational law in Theorem 5 , it is easy to deduce the ICD of $f_{2}\left(\zeta_{2}\right)$ where the function $f_{2}$ is defined as

$f_{2}(t)=5-2 t \vee 1$.

Since $f_{2}$ is decreasing, in the light of Theorem 5 , the ICD of $f_{2}\left(\zeta_{2}\right)$ is derived as (see Figure 12)

$$
\begin{aligned}
\psi_{f_{2}}^{-1}(\delta)= & f_{2}\left(\psi_{2}^{-1}(1-\delta)\right) \\
= & \begin{cases}4 \delta-1, & \text { if } \quad 0 \leq \delta<\frac{1}{2} \\
{[1,3],} & \text { if } \quad \delta=\frac{1}{2} \\
8 \delta-1, & \text { if } \quad \frac{1}{2}<\delta \leq \frac{5}{8} \\
4, & \text { if } \quad \frac{5}{8}<\delta \leq 1 .\end{cases}
\end{aligned}
$$

Example 14 Let $\zeta_{1}$ and $\zeta_{2}$ be independent trapezoidal fuzzy numbers denoted by $\mathcal{T}(2,4,2,3)_{L R}$ and $\mathcal{T}(1,2,2,1)_{L R}$ with the ICDs $\psi_{1}^{-1}$ and $\psi_{2}^{-1}$, respectively. As the function $f\left(t_{1}, t_{2}\right)=f_{1}\left(t_{1}\right)+$ $f_{2}\left(t_{2}\right)$ with $f_{1}$ and $f_{2}$ defined in Eqs. (20) and (24) respectively is increasing regarding $t_{1}$ and decreasing regarding $t_{2}$, in accordance with Theorem 5 , the ICD of $\zeta=f\left(\zeta_{1}, \zeta_{2}\right)=f_{1}\left(\zeta_{1}\right)+f_{2}\left(\zeta_{2}\right)$ is obtained as

$$
\psi^{-1}(\delta)=f_{1}\left(\psi_{1}^{-1}(\delta)\right)+f_{2}\left(\psi_{2}^{-1}(1-\delta)\right) .
$$




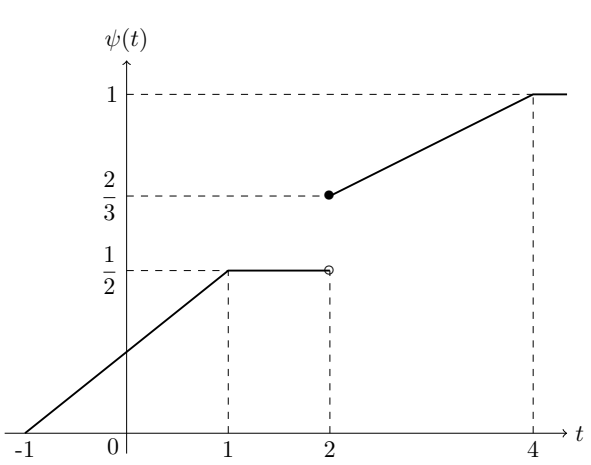

Fig. 10 The credibility distribution of $f_{1}\left(\zeta_{1}\right)$ in Example 11

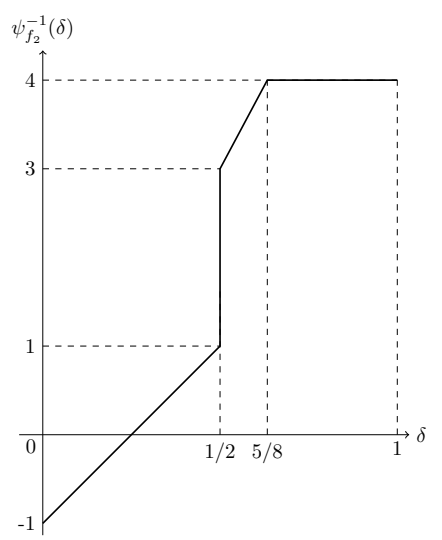

Fig. 12 The ICD of $f_{2}\left(\zeta_{2}\right)$ in Example 13

Then, on account of Eqs. (23) and (25), we can obtain that

$$
\psi^{-1}(\delta)=\left\{\begin{array}{l}
8 \delta-2, \text { if } 0 \leq \delta<\frac{1}{2} \\
{[2,5], \quad \text { if } \delta=\frac{1}{2}} \\
8 \delta+1, \text { if } \frac{1}{2}<\delta \leq \frac{5}{8} \\
6, \quad \text { if } \frac{5}{8}<\delta \leq \frac{2}{3} \\
6 \delta+2, \text { if } \frac{2}{3}<\delta \leq 1,
\end{array}\right.
$$

as depicted in Figure 13.

\subsection{Expected value}

Expected value $(\mathrm{EV})$ is the mean value of all possible values of a fuzzy variable in the sense of fuzzy measure. Based on the EV of a fuzzy variable defined by Liu and Liu Liu and Liu (2002), a calculation formula of the EV of an LR-FI is presented.

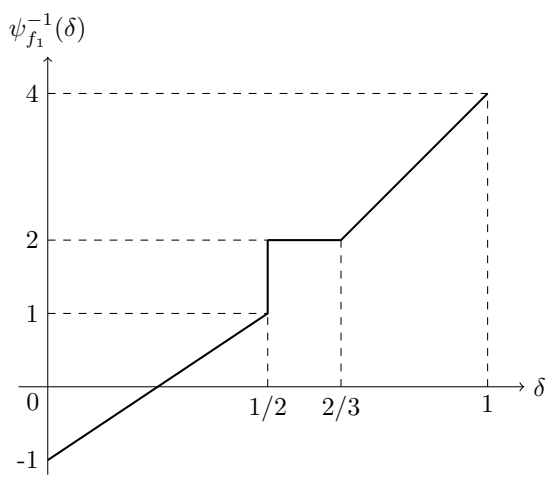

Fig. 11 The ICD of $f_{1}\left(\zeta_{1}\right)$ in Example 12

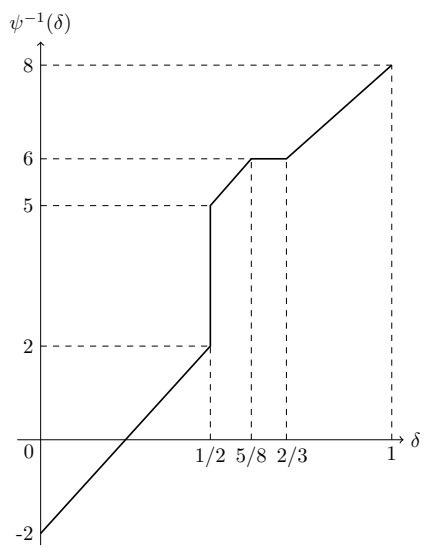

Fig. 13 The ICD of $f\left(\zeta_{1}, \zeta_{2}\right)$ in Example 14

Definition 6 (Liu and Liu 2002) If $\zeta$ is a fuzzy variable, then its $E V$ is defined as

$$
E[\zeta]=\int_{0}^{+\infty} \operatorname{Cr}\{\zeta \geq t\} \mathrm{d} t-\int_{-\infty}^{0} \operatorname{Cr}\{\zeta \leq t\} \mathrm{d} t
$$

suppose that at least one of the two integrals is finite.

Theorem 6 If the EV of the LR-FI $\zeta$ exists, then

$E[\zeta]=\int_{0}^{1} \psi^{-1}(\delta) \mathrm{d} \delta$,

where $\psi^{-1}$ is the ICD of $\zeta$.

Proof: The proof is provided in Appendix E. 
Example 15 Let $\zeta$ be $\mathcal{T}(\underline{c}, \bar{c}, \rho, \sigma)_{L R}$. Its $\mathrm{EV}$ is

$$
\begin{aligned}
E[\zeta]= & \int_{0}^{0.5}(2 \rho \delta+\underline{c}-\rho) \mathrm{d} \delta \\
& +\int_{0.5}^{1}(2 \sigma \delta+\bar{c}-\delta) \mathrm{d} \delta \\
= & \frac{2 \underline{c}+2 \bar{c}+\sigma-\rho}{4} .
\end{aligned}
$$

Following from Theorem 5 and Theorem 6, a theorem that can be able to calculate EVs of monotone functions with regard to regular LR-FIs is proposed.

Theorem 7 Let $\zeta_{1}, \zeta_{2}, \cdots, \zeta_{n}$ be independent regular LR-FIs with ICDs $\psi_{1}^{-1}, \psi_{2}^{-1}, \cdots, \psi_{n}^{-1}$, respectively. If the continuous function $f\left(t_{1}, t_{2}\right.$, $\left.\cdots, t_{n}\right)$ is increasing regarding $t_{1}, t_{2}, \cdots, t_{k}$ and decreasing regarding $t_{k+1}, t_{k+2}, \cdots, t_{n}$, then the $E V$ of the $L R-F I \zeta=f\left(\zeta_{1}, \zeta_{2}, \cdots, \zeta_{n}\right)$ is

$$
\begin{aligned}
E[\zeta]= & \int_{0}^{1} f\left(\psi_{1}^{-1}(\delta), \cdots, \psi_{k}^{-1}(\delta), \psi_{k+1}^{-1}(1-\delta),\right. \\
& \left.\cdots, \psi_{n}^{-1}(1-\delta)\right) \mathrm{d} \delta .
\end{aligned}
$$

Proof: On the basis of Theorem 5, we can derive that the ICD of $\zeta=f\left(\zeta_{1}, \zeta_{2}, \cdots, \zeta_{n}\right)$ is $f\left(\psi_{1}^{-1}(\delta), \cdots, \psi_{k}^{-1}(\delta), \psi_{k+1}^{-1}(1-\delta), \cdots, \psi_{n}^{-1}(1-\right.$ $\delta))$. Then by using Theorem 6 , we obtain Eq. (31).

Example 16 Let us consider the EV of $\zeta=f\left(\zeta_{1}\right.$, $\left.\zeta_{2}\right)=f_{1}\left(\zeta_{1}\right)+f_{2}\left(\zeta_{2}\right)$ in Example 13, whose ICD is $f_{1}\left(\psi_{1}^{-1}(\delta)\right)+f_{2}\left(\psi_{2}^{-1}(1-\delta)\right)$ in Eq. (27). Then according to Theorem 7 , the $\mathrm{EV}$ of $\zeta=f\left(\zeta_{1}, \zeta_{2}\right)$ is

$$
\begin{aligned}
E[\zeta]= & \int_{0}^{1}\left(f_{1}\left(\psi_{1}^{-1}(\delta)\right)+f_{2}\left(\psi_{2}^{-1}(1-\delta)\right)\right) \mathrm{d} \delta \\
= & \int_{0}^{\frac{1}{2}}(8 \delta-2) \mathrm{d} \delta+\int_{\frac{1}{2}}^{\frac{5}{8}}(8 \delta+2) \mathrm{d} \delta \\
& +\int_{\frac{5}{8}}^{\frac{2}{3}} 6 \mathrm{~d} \delta+\int_{\frac{2}{3}}^{1}(6 \delta+2) \mathrm{d} \delta \\
= & \frac{157}{48} .
\end{aligned}
$$

\section{Fuzzy programming}

Fuzzy programming is a type of mathematical models to address optimization problems involving fuzzy parameters, which has been studied by many researchers from different points of view (see Liu 1998; Liu and Liu 2002; Li$\mathrm{u}$ and Iwamura 1998a,b; Zhou et al. 2016). In this section, we discuss the fuzzy CCP model in Zhou et al. (2016) containing monotone but not necessarily strictly monotone objective and constraint functions with regular LR-FIs and then develop a solution framework.

4.1 Fuzzy CCP and its equivalent crisp model

Suppose that $\boldsymbol{t}$ is a decision vector, $\boldsymbol{\zeta}=\left(\zeta_{1}, \zeta_{2}\right.$, $\left.\cdots, \zeta_{n}\right)$ is an $n$-dimensional fuzzy vector, $f(\boldsymbol{t}, \boldsymbol{\zeta})$ is the objective function, and $h_{v}(\boldsymbol{t}, \boldsymbol{\zeta})$ is the constraint function for $v=1,2, \cdots, w$. Owing to the fuzziness of the objective function $f(\boldsymbol{t}, \boldsymbol{\zeta})$, it is hard to be minimized directly. As an alternative way, it is quite natural to minimize its $\mathrm{EV}$, i.e., $E[f(\boldsymbol{t}, \boldsymbol{\zeta})]$. In addition, as to the fuzzy constraints $h_{v}(\boldsymbol{t}, \boldsymbol{\zeta}) \leq 0$ for $v=1,2, \cdots, w$, since there is no deterministic feasible set defined by them, Liu and Iwamura (1998a) suggested that it should be desirable that the solutions satisfy the fuzzy constraints at a predetermined confidence level $\delta_{v}$ for $v=1,2, \cdots, w$, that is,

$\operatorname{Cr}\left\{h_{v}(\boldsymbol{t}, \boldsymbol{\zeta}) \leq 0\right\} \geq \delta_{v}, \quad v=1,2, \cdots, w$.

In this way, a fuzzy CCP model to minimize the EV of objective function under a series of chance constraints was constructed by Zhou et al. (2016) as

$$
\left\{\begin{array}{l}
\min _{\boldsymbol{t}} E[f(\boldsymbol{t}, \boldsymbol{\zeta})] \\
\text { subject to: } \\
\quad \operatorname{Cr}\left\{h_{v}(\boldsymbol{t}, \boldsymbol{\zeta}) \leq 0\right\} \geq \delta_{v}, \quad v=1,2, \cdots, w .
\end{array}\right.
$$

When fuzzy parameters in the fuzzy CCP model (33) are regular LR-FIs, and the objective and constraint functions are both continuous and monotone with regard to these fuzzy parameters, model (33) can be converted to a deterministic counterpart, which is verified in the following theorem.

Theorem 8 Assume that the function $f\left(\boldsymbol{t}, \zeta_{1}\right.$, $\left.\zeta_{2}, \cdots, \zeta_{n}\right)$ is continuous and increasing regarding $\zeta_{1}, \zeta_{2}, \cdots, \zeta_{k}$ and decreasing regarding $\zeta_{k+1}$, $\zeta_{k+2}, \cdots, \zeta_{n}$, and the functions $h_{v}\left(\boldsymbol{t}, \zeta_{1}, \zeta_{2}, \cdots\right.$, $\left.\zeta_{n}\right)$ are increasing regarding $\zeta_{1}, \zeta_{2}, \cdots, \zeta_{k_{v}}$ and decreasing regarding $\zeta_{k_{v}+1}, \zeta_{k_{v}+2}, \cdots, \zeta_{n}$ for $v=$ 
$1,2, \cdots, w$. If $\zeta_{1}, \zeta_{2}, \cdots, \zeta_{n}$ are independent regular LR-FIs, then model (33) can be converted to the following crisp equivalent

$$
\left\{\begin{array}{l}
\min _{\boldsymbol{t}} \int_{0}^{1} f\left(\boldsymbol{t}, \psi_{1}^{-1}(\delta), \cdots, \psi_{k}^{-1}(\delta), \psi_{k+1}^{-1}(1-\delta),\right. \\
\left.\quad \cdots, \psi_{n}^{-1}(1-\delta)\right) \mathrm{d} \delta \\
\text { subject to: } \\
\quad h_{v}\left(\boldsymbol{t}, \psi_{1}^{-1}\left(\delta_{v}\right), \cdots, \psi_{k_{v}}^{-1}\left(\delta_{v}\right), \psi_{k_{v}+1}^{-1}\left(1-\delta_{v}\right),\right. \\
\left.\quad \cdots, \psi_{n}^{-1}\left(1-\delta_{v}\right)\right) \leq 0, \\
v=1,2, \cdots, w
\end{array}\right.
$$

where $\psi_{i}^{-1}$ is the ICD of $\zeta_{i}$ for $i=1,2, \cdots, n$.

Proof: The proof is provided in Appendix F.

\subsection{Solution methods}

It is worth noting that there exists an integral in the objective function of model (34), which means that the fuzzy model (33) cannot be solved directly by well-developed software packages after translation. In order to solve model (33), Liu (2002) designed a hybrid intelligent algorithm (HIA) by combining stochastic discretization algorithm (SDA), neural network and genetic algorithm. However, Li (2015) and Liu et al. (2020) pointed out that SDA has poor performance both on accuracy and computational time over simulating the EV. Liu et al. (2020) subsequently proposed a numericalintegral based algorithm, but it is not applicable to monotone but not necessarily strictly monotone functions with regard to regular LRFIs. Thus this paper proposes a new numerical integration algorithm (NIA) to fill the gap.

With regard to the basic principle of NIA for simulating $E[f(\boldsymbol{t}, \boldsymbol{\zeta})]$, on account of Theorem 8, we know that $E[f(\boldsymbol{t}, \boldsymbol{\zeta})]$ is an integration of function $f\left(\boldsymbol{t}, \psi_{1}^{-1}(\delta), \cdots, \psi_{k}^{-1}(\delta), \psi_{k+1}^{-1}(1-\delta)\right.$, $\left.\cdots, \psi_{n}^{-1}(1-\delta)\right)$. Based on the definition of definite integral in mathematics, we partition the closed interval $[0,1]$ into $S$ equal parts and take the value of the right of each equal part as the integration variable, that is, $\delta=s / S$ for $s=1,2, \cdots, S$. When the number of integration points, $S$, is set to be sufficiently large, we can obtain that

$$
\begin{array}{r}
E[f(\boldsymbol{t}, \boldsymbol{\zeta})] \approx \sum_{s=1}^{S} f\left(\boldsymbol{t}, \psi_{1}^{-1}(s / S), \cdots, \psi_{k}^{-1}(s / S),\right. \\
\left.\psi_{k+1}^{-1}(1-s / S), \cdots, \psi_{n}^{-1}(1-s / S)\right) / S .
\end{array}
$$

The NIA is given as Algorithm 1.

\section{Algorithm 1: (NIA)}

Step 1. Initialize the integration points $S$; $E=0 ; s=1$.

Step 2. Calculate $\gamma_{i}=\psi_{i}^{-1}(s / S)$ for each $1 \leq$ $i \leq k$ and $\gamma_{i}=\psi_{i}^{-1}(1-s / S)$ for $k+1$ $\leq i \leq n$ based on Eq. (17).

Step 3. Update $E=E+f\left(\boldsymbol{t}, \gamma_{1}, \gamma_{2}, \ldots, \gamma_{n}\right) / S$ and $s=s+1$.

Step 4. If $s \leq S$, go to Step 2. Otherwise, return the $E$.

To illustrate the performance of NIA on accuracy and efficiency, comparisons between NI$\mathrm{A}$ and SDA for simulating the EV of monotone functions over some numerical experiments of an example are conducted.

Example 17 Consider two continuous and monotone functions $f_{1}\left(\zeta_{1}, \zeta_{2}\right)=5 \wedge \zeta_{1}-1 \vee \zeta_{2}$ and $f_{2}\left(\zeta_{1}, \zeta_{2}\right)=5 \wedge \zeta_{1} /\left(1 \vee \zeta_{2}\right)$, in which fuzzy intervals $\zeta_{1} \sim(2,4,2,3)_{L R}$ and $\zeta_{2} \sim(1,2,2,1)_{L R}$ are set to regular LR-FIs appearing in Examples 1-3 successively.

For each case, after using SDA and NIA to calculate EVs of the function respectively, the experimental results covering exact value, simulation value and running time are all listed in Table 1. To facilitate comparing the differences between the simulation values obtained by two algorithms and the exact value obtained by Matlab, a parameter named Error is introduced, which is derived from the formula $\mid$ simulation value - exact value / / exact value $\times 100 \%$.

From Table 1, it can be seen that there are slight differences with Errors no more than $6 \%$ between the EVs obtained by two algorithms and the exact values. Compared with SDA, NI$\mathrm{A}$ is more reliable and stable in the accuracy of solutions and solution efficiency. More specifically, the largest error for SDA is up to $5.7871 \%$, while the errors for NIA are all not more than $0.05 \%$. On the other hand, the running time of 
Table 1 Comparison between SDA and NIA

\begin{tabular}{ccccccc}
\hline Algorithm & \multicolumn{2}{c}{$\mathcal{T}(\underline{c}, \bar{c}, \rho, \sigma)_{L R}$} & \multicolumn{2}{c}{$\mathcal{A}(\underline{c}, \bar{c}, \rho, \sigma)_{L R}$} & \multicolumn{2}{c}{$\mathcal{B}(\underline{c}, \bar{c}, \rho, \sigma)_{L R}$} \\
\hline SDA & $f_{1}$ & $f_{2}$ & $f_{1}$ & $f_{2}$ & $f_{1}$ & $f_{2}$ \\
Exact value & 1.1667 & 2.6331 & 0.9815 & 2.6159 & 1.1481 & 2.6804 \\
Simulation value & 1.2217 & 2.6357 & 1.0383 & 2.6193 & 1.2076 & 2.6825 \\
Error (\%) & 4.7142 & 0.0987 & 5.7871 & 0.1300 & 5.1824 & 0.0783 \\
Running time (s) & 0.176 & 0.181 & 0.173 & 0.177 & 0.168 & 0.174 \\
\hline NIA & $f_{1}$ & $f_{2}$ & $f_{1}$ & $f_{2}$ & $f_{1}$ & $f_{2}$ \\
Exact value & 1.1667 & 2.6331 & 0.9815 & 2.6159 & 1.1481 & 2.6804 \\
Simulation value & 1.1671 & 2.6331 & 0.9819 & 2.6159 & 1.1485 & 2.6804 \\
Error (\%) & 0.0343 & 0.0000 & 0.0408 & 0.0000 & 0.0348 & 0.0000 \\
Running time (s) & 0.001 & 0.001 & 0.001 & 0.001 & 0.001 & 0.001 \\
\hline
\end{tabular}

SDA is more than 160 times than NIA's. Overall, NIA outperforms SDA and is probably able to get an accurate value in a relatively short time.

Based on the above analyses, we embed NIA used for simulating $E[f(\boldsymbol{t}, \boldsymbol{\zeta})]$ into a classical genetic algorithm, thereby formulating a new algorithm (NIA-GA) to dispose of model (34), whose performance will be compared with HIA algorithm and evaluated on a set of numerical experiments from a purchasing planning problem in the following section.

\subsection{Numerical example}

Provided that there is a dealer selling $n$ types of products, he would like to determine the optimal order quantity to satisfy customer demands for products with the aim of maximizing the total profit. In order to have a better understanding for this problem, some assumptions are given as follows and some relevant notations are shown in Table 2 where the parameter values are summarized in Table 3.

\section{Assumptions}

1. The customer demands are uncertain and characterized by regular LR-FIs.

2. Any leftover inventory can be salvaged at a unit value, which is lower than the selling price.

3. The total cost of purchasing products from supplier is not more than budget.

According to the assumptions and notation$\mathrm{s}$, the total procurement cost, total opportunity loss and the total profit are

$C(\boldsymbol{t})=\sum_{i=1}^{n} c_{i} t_{i}$
$\left.S(\boldsymbol{t}, \boldsymbol{\zeta})=\sum_{i=1}^{n} s_{i}\left(\zeta_{i}-\zeta_{i} \wedge t_{i}\right)\right)$

and

$\Pi(\boldsymbol{t}, \boldsymbol{\zeta})=\sum_{i=1}^{n}\left[\left(p_{i}-v_{i}\right)\left(\zeta_{i} \wedge t_{i}\right)+\left(v_{i}-c_{i}\right) t_{i}\right]$

respectively.

Assuming that the total budget on the procurement is $C^{0}$ and the biggest opportunity loss the dealer can undertake is $S^{0}$, then it follows from the idea of fuzzy CCP model (33) that a fuzzy CCP model for this problem is constructed as

$$
\left\{\begin{array}{l}
\max E\left[\sum_{i=1}^{n}\left[\left(p_{i}-v_{i}\right)\left(\zeta_{i} \wedge t_{i}\right)+\left(v_{i}-c_{i}\right) t_{i}\right]\right] \\
\text { subject to: } \\
\sum_{i=1}^{n} c_{i} t_{i} \leq C^{0} \\
\left.\operatorname{Cr}\left\{\sum_{i=1}^{n} s_{i}\left(\zeta_{i}-\zeta_{i} \wedge t_{i}\right)\right) \leq S^{0}\right\} \geq \delta_{0} \\
t_{i} \geq 0, \quad i=1,2, \cdots, n .
\end{array}\right.
$$

Apparently, both the total profit $\Pi(\boldsymbol{t}, \boldsymbol{\zeta})$ and the total opportunity loss $S(\boldsymbol{t}, \boldsymbol{\zeta})$ are increasing but not strictly increasing with respect to $\zeta_{i}$. Here we assume that the retailer has three type$\mathrm{s}$ of the products, and other parameter values are all summarized in Table 3. Then based on Theorem 8, a deterministic programming model 
Table 2 Notations

\begin{tabular}{ll}
\hline Decision variable & \\
$t_{i}$ & order quantity of the $i$ th type of products, $i=1,2, \cdots, n$ \\
Parameters & purchasing cost of the $i$ th type of products, $i=1,2, \cdots, n$ \\
$c_{i}$ & selling price of the $i$ th type of products, $i=1,2, \cdots, n$ \\
$p_{i}$ & salvage value of the $i$ th type of products, $i=1,2, \cdots, n$ \\
$v_{i}$ & opportunity loss of the $i$ th type of products, $i=1,2, \cdots, n$ \\
$s_{i}$ & customer demand of $i$ th type of products, $i=1,2, \cdots, n$ \\
$\zeta_{i}$ & total purchasing cost, where $\boldsymbol{t}=\left(t_{1}, t_{2}, \ldots, t_{n}\right)$ \\
$C(\boldsymbol{t})$ & total opportunity loss, where $\boldsymbol{t}=\left(t_{1}, t_{2}, \ldots, t_{n}\right), \boldsymbol{\zeta}=\left(\zeta_{1}, \zeta_{2}, \cdots, \zeta_{n}\right)$ \\
$S(\boldsymbol{t}, \boldsymbol{\zeta})$ & total profit, where $\boldsymbol{t}=\left(t_{1}, t_{2}, \ldots, t_{n}\right), \boldsymbol{\zeta}=\left(\zeta_{1}, \zeta_{2}, \cdots, \zeta_{n}\right)$ \\
$\Pi(\boldsymbol{t}, \boldsymbol{\zeta})$ &
\end{tabular}

can be obtained as

$$
\left\{\begin{array}{l}
\max \int_{0}^{1}\left[40\left(\psi_{1}^{-1}(\delta) \wedge t_{1}\right)+20\left(\psi_{2}^{-1}(\delta) \wedge t_{2}\right)\right. \\
\left.+80\left(\psi_{3}^{-1}(\delta) \wedge t_{3}\right)\right] \mathrm{d} \delta-10 t_{1}-10 t_{2}-20 t_{3} \\
\text { subject to: } \\
\quad 50 t_{1}+40 t_{2}+70 t_{3} \leq C^{0} \\
\quad 30\left(\psi_{1}^{-1}\left(\delta_{0}\right)-\psi_{1}^{-1}\left(\delta_{0}\right) \wedge t_{1}\right)+10\left(\psi_{2}^{-1}\left(\delta_{0}\right)\right. \\
\left.-\psi_{2}^{-1}\left(\delta_{0}\right) \wedge t_{2}\right)+60\left(\psi_{3}^{-1}\left(\delta_{0}\right)-\psi_{3}^{-1}\left(\delta_{0}\right)\right. \\
\left.\wedge t_{3}\right) \leq S^{0} \\
t_{i} \geq 0, \quad i=1,2,3,
\end{array}\right.
$$

where $\psi_{i}^{-1}$ is the ICD of $\zeta_{i}$, which can be derived from Theorem 1.

Afterward, 20 test problems are generated by increasing $C^{0}$ from 61000 to 70000 with an increase of 1000 and decreasing $S^{0}$ from 6500 to 2000 with a decrease of 500 simultaneously under the confidence level fixed at 0.8 and 0.9 , respectively. For each problem, HIA and NIA-GA are run accordingly to solve the corresponding models. Considering the randomness of results obtained by metaheuristic algorithm$\mathrm{s}$, we implement each test problem for 10 times and then select the optimal solution with the best target value as the final solution. Then the optimal solutions, the corresponding target values, $E\left[\Pi\left(\boldsymbol{t}^{*}, \boldsymbol{\zeta}\right)\right]$, and the average time for running 10 times are shown in Table 4. Moreover, in accordance with the poor performance of $\mathrm{F}$ $\mathrm{S}$ in HIA as illustrated in Section 3.2, a new EV of profit, $E\left[\Pi\left(\boldsymbol{t}^{*}, \boldsymbol{\zeta}\right)\right]^{*}$, is computed by substituting the optimal solution acquired by HIA into NIA, which is listed in the last column of
Table 4. So, it makes sense to judge the quality of the optimal solutions obtained by HIA and NIA-GA by comparing $E\left[\Pi\left(\boldsymbol{t}^{*}, \boldsymbol{\zeta}\right)\right]^{*}$ with $E\left[\Pi\left(\boldsymbol{t}^{*}, \boldsymbol{\zeta}\right)\right]$ in last third column of Table 4 . Furthermore, for the sake of visualising the differences better, the target values, $E\left[\Pi\left(\boldsymbol{t}^{*}, \boldsymbol{\zeta}\right)\right]$ obtained by two solution methods and $E\left[\Pi\left(\boldsymbol{t}^{*}, \boldsymbol{\zeta}\right)\right]^{*}$ in Table 4 are plotted in Figures 14(a) and 14(b).

From Table 4, it can be seen that NIA-GA possesses an outstanding advantage over HIA in terms of running time. Concretely, the running time of NIA-GA is almost one hundred times faster than that of HIA. In the meantime, as for the quality of solutions found by two methods, we can conclude that the solutions derived by NIA-GA are all better than those obtained by HIA, since it can be observed from Figures $14(\mathrm{a})$ and $14(\mathrm{~b})$ that $E\left[\Pi\left(\boldsymbol{t}^{*}, \boldsymbol{\zeta}\right)\right]$ derived by NIA-GA are all larger than $E\left[\Pi\left(\boldsymbol{t}^{*}, \boldsymbol{\zeta}\right)\right]^{*}$, and the maximum relative deviation (calculated by formula $\left|E\left[\Pi\left(\boldsymbol{t}^{*}, \boldsymbol{\zeta}\right)\right]-E\left[\Pi\left(\boldsymbol{t}^{*}, \boldsymbol{\zeta}\right)\right]^{*}\right| / E\left[\Pi\left(\boldsymbol{t}^{*}\right.\right.$ $\boldsymbol{\zeta})] \times 100 \%)$ is up to $3.12 \%$ for No.4 test problem. Additionally, we can also see the obvious difference between $E\left[\Pi\left(\boldsymbol{t}^{*}, \boldsymbol{\zeta}\right)\right]$ from HIA and $E\left[\Pi\left(\boldsymbol{t}^{*}, \boldsymbol{\zeta}\right)\right]^{*}$ recalculated by NIA, and the maximum relative deviation (calculated by formula $\left|E\left[\Pi\left(\boldsymbol{t}^{*}, \boldsymbol{\zeta}\right)\right]-E\left[\Pi\left(\boldsymbol{t}^{*}, \boldsymbol{\zeta}\right)\right]^{*}\right| / E\left[\Pi\left(\boldsymbol{t}^{*}, \boldsymbol{\zeta}\right)\right]^{*} \times$ $100 \%$ ) reaches $0.81 \%$ for No.10 test problem.

In summary, compared with HIA, NIA-GA not only has excellent performance on running time but also can obtain a better target value, mainly because NIA-GA reduces the number of fuzzy simulation on constraint functions, and NIA for the computation of EV of total profit $E\left[\Pi\left(\boldsymbol{t}^{*}, \boldsymbol{\zeta}\right)\right]$ is more precise than FS. The results also imply that our solution method could provide the retailer with a better reference to 
Table 3 Parameter values of the numerical example

\begin{tabular}{cccc}
\hline Parameters & $i=1$ & $i=2$ & $i=3$ \\
\hline$\zeta_{i}$ & $\mathcal{T}(380,400,20,30)_{L R}$ & $\mathcal{T}(470,510,10,20)_{L R}$ & $\mathcal{T}(300,360,50,40)_{L R}$ \\
$p_{i}$ & 80 & 50 & 130 \\
$c_{i}$ & 50 & 40 & 70 \\
$v_{i}$ & 40 & 30 & 50 \\
$s_{i}$ & 30 & 10 & 60 \\
\hline
\end{tabular}

Table 4 Experimental results

\begin{tabular}{|c|c|c|c|c|c|c|c|c|}
\hline \multirow[b]{2}{*}{ No. } & \multirow[b]{2}{*}{$\delta_{0}$} & \multirow[b]{2}{*}{$C^{0}$} & \multirow[b]{2}{*}{$S^{0}$} & \multicolumn{2}{|c|}{ HIA } & \multicolumn{2}{|c|}{ NIA-GA } & \multirow[b]{2}{*}{$E\left[\Pi\left(\boldsymbol{t}^{*}, \boldsymbol{\zeta}\right)\right]^{*}$} \\
\hline & & & & $E\left[\Pi\left(\boldsymbol{t}^{*}, \boldsymbol{\zeta}\right)\right]$ & $\begin{array}{l}\text { Running } \\
\text { time (s) }\end{array}$ & $E\left[\Pi\left(\boldsymbol{t}^{*}, \boldsymbol{\zeta}\right)\right]$ & $\begin{array}{c}\text { Running } \\
\text { time }(\mathrm{s})\end{array}$ & \\
\hline 1 & 0.9 & 61000 & 6500 & 33366.09 & 2781.12 & 33548.43 & 27.03 & 33188.40 \\
\hline 2 & 0.9 & 62000 & 6000 & 33207.61 & 2926.56 & 33850.47 & 26.72 & 33093.59 \\
\hline 3 & 0.9 & 63000 & 5500 & 33502.66 & 3005.67 & 34088.54 & 26.49 & 33389.68 \\
\hline 4 & 0.9 & 64000 & 5000 & 33369.17 & 3043.23 & 34280.58 & 26.74 & 33211.55 \\
\hline 5 & 0.9 & 65000 & 4500 & 33980.26 & 3015.78 & 34452.78 & 27.26 & 33831.01 \\
\hline 6 & 0.9 & 66000 & 4000 & 34354.63 & 3054.89 & 34515.35 & 27.44 & 34131.97 \\
\hline 7 & 0.9 & 67000 & 3500 & 34731.30 & 3025.69 & 34525.38 & 28.03 & 34475.98 \\
\hline 8 & 0.9 & 68000 & 3000 & 34745.76 & 3056.13 & 34525.23 & 27.39 & 34514.03 \\
\hline 9 & 0.9 & 69000 & 2500 & 34770.69 & 3070.93 & 34525.23 & 27.60 & 34513.42 \\
\hline 10 & 0.9 & 70000 & 2000 & 34772.15 & 3064.36 & 34525.39 & 27.55 & 34493.91 \\
\hline 11 & 0.8 & 61000 & 6500 & 33280.12 & 2876.26 & 33560.44 & 26.44 & 33112.88 \\
\hline 12 & 0.8 & 62000 & 6000 & 33478.67 & 2906.11 & 33850.47 & 26.62 & 33280.79 \\
\hline 13 & 0.8 & 63000 & 5500 & 33790.93 & 3006.77 & 34050.07 & 27.08 & 33550.23 \\
\hline 14 & 0.8 & 64000 & 5000 & 33907.88 & 2930.04 & 34256.08 & 26.93 & 33661.15 \\
\hline 15 & 0.8 & 65000 & 4500 & 34602.57 & 3002.46 & 34417.79 & 26.72 & 34361.39 \\
\hline 16 & 0.8 & 66000 & 4000 & 34748.72 & 3012.32 & 34515.35 & 27.36 & 34491.82 \\
\hline 17 & 0.8 & 67000 & 3500 & 34760.56 & 2960.77 & 34525.38 & 27.20 & 34490.77 \\
\hline 18 & 0.8 & 68000 & 3000 & 34719.65 & 3077.22 & 34525.72 & 27.34 & 34491.92 \\
\hline 19 & 0.8 & 69000 & 2500 & 34750.27 & 3077.74 & 34525.23 & 27.37 & 34505.39 \\
\hline 20 & 0.8 & 70000 & 2000 & 34743.07 & 3054.82 & 34525.38 & 27.40 & 34478.86 \\
\hline
\end{tabular}

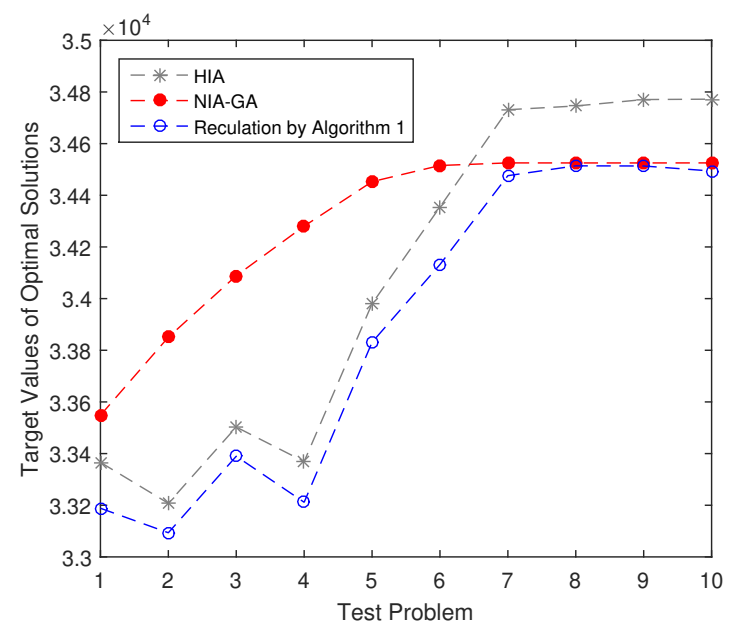

(a) $\delta_{0}=0.9$

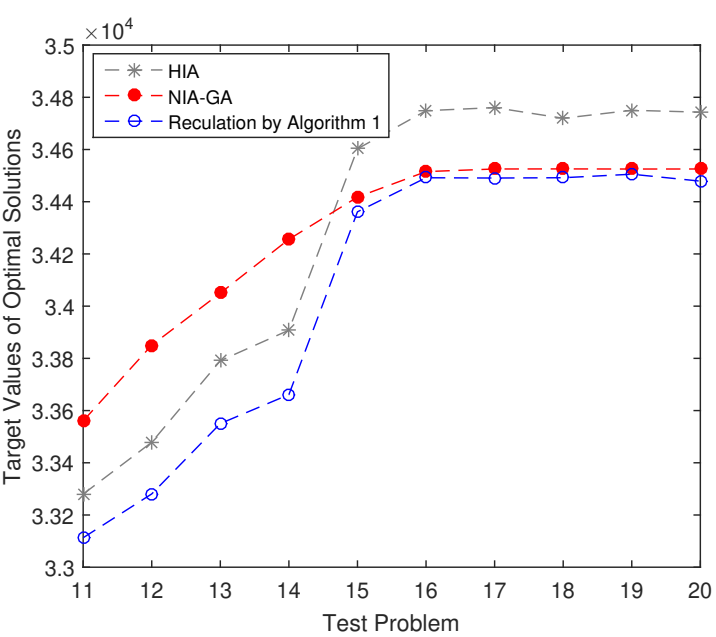

(b) $\delta_{0}=0.8$

Fig. 14 Comparison of target valules for different algorithms. 
place the order so as to reduce the risk of the overstocking or shortage of products.

\section{Conclusion}

Fuzzy arithmetic is of great importance as an advanced tool to be used in fuzzy optimization and control theory. In this research field, Zhou et al. Zhou et al. (2016) proposed an operational law to exactly calculate the credibility distribution of strictly monotone functions regarding regular LR fuzzy numbers, which facilitates the development of fuzzy arithmetic both in theory and application. Although the operational law is rather useful to handle many fuzzy optimization problems, restrictions on the strictly monotone functions and regular LR fuzzy numbers block its applications to some problems modeled by monotone functions with LR fuzzy intervals, such as the classical newsvendor problem with fuzzy demands represented by trapezoidal fuzzy numbers. Thus, this paper aims at generalizing the operational law in Zhou et al. (2016) and then explored the generalized operational law's applications to fuzzy arithmetic and fuzzy optimization problems.

The main findings of this study are as follows. Firstly, the ICD of an LR-FI in view of the credibility measure was defined and accordingly its calculation formula was suggested. Following that, some equivalent conditions of the regular LR-FI were proved. Next, an extensive operational law on exactly calculating ICDs of monotone functions with regard to regular LR-FIs was proposed. Then an equivalent formula for calculating the EV of an LR-FI and a theorem for calculating the EVs of monotone functions were proposed. Subsequently, a solution strategy for the fuzzy CCP with monotone functions of regular LR-FIs was formulated, where the fuzzy model was translated into a crisp equivalent first and then a new heuristic algorith$m$ called NIA-GA which integrates NIA with a standard GA was devised. Finally, the proposed solution method was applied to a purchasing planning problem and its performance was demonstrated by comparing with HIA over a set of numerical experiments. The computational results reveal that our method outperforms HIA in both solution accuracy and efficiency and could also assist the decision-maker to determine a suitable order quantity under uncertain environment to maximize the expected profit.

For future work, the theoretical findings in this paper can be applied to deal with many other optimization problems under the fuzzy environment such as the project scheduling problem and the reliability optimization problems. On the other hand, we can extend this study further from the continuous functions to the noncontinuous functions in theory so that more practical problems modeled by the noncontinuous functions of fuzzy variables can be worked out easily.

\section{Compliance with Ethical Standards}

Funding: This work was supported in part by grants from the National Natural Science Foundation of China (Grant No. 71872110).

Conflict of interest: The authors declare that they have no conflict of interest to this work.

Authors' contributions: Conceptualization: M. Zhao; Formal analysis: M. Zhao; Methodology: M. Zhao; Writing - original draft: M. Zhao and Y. Han; Writing - review and editing: J. Zhou; Funding acquisition: J. Zhou; Project administration: J. Zhou; Resources: J. Zhou; Software: Y. Han; Supervision: J. Zhou; Validation: Y. Han; Visualization: Y. Han.

Ethical approval: This article does not contain any studies with human participants or animals performed by any of the authors.

\section{References}

Abbasi F, Allahviranloo T (2019) New operations on pseudo-octagonal fuzzy numbers and its application. Soft Comput 23(19): 97619776

Ban AI, Coroianu L, Khastan A (2016) Conditioned weighted L-R approximations of fuzzy numbers. Fuzzy Sets Syst 283: 56-82

Büyüközkan G, Feyzioglu O, Göcer F (2018) Selection of sustainable urban transportation alternatives using an integrated intuitionistic fuzzy Choquet integral approach. Transport Res Part D-Transport Environ 58: 186-207

Chou CC (2003) The canonical representation of multiplication operation on triangular 
fuzzy numbers. Comput Math Appl 45(1011): $1601-1610$

Dombi J, Gyorbiro N (2006) Addition of sigmoid-shaped fuzzy intervals using the Dombi operator and infinite sum theorems. Fuzzy Sets Syst 157(7): 952-963

Dubois D, Prade H (1978) Operations on fuzzy numbers. Int J Syst Sci 9(6): 613-626.

Dubois D, Prade H (1979) Fuzzy real algebra: some results. Fuzzy Sets Syst 2(4): 327-34

Dubois D, Prade H (1988) Possibility Theory. Plenum Press, New York

Goetschel R, Voxman W (1986) Elementary fuzzy calculus. Fuzzy Sets Syst 18(1): 31-43

Hong DH, Hwang C (1997) A T-sum bound of LR-fuzzy numbers. Fuzzy Sets Syst 91(2): 239-252

Hong DH (2007) T-sum of bell-shaped fuzzy intervals. Fuzzy Sets Syst 158(7): 739-746

Hong DH, Hwang C, Kim KT (2007) T-sum of sigmoid-shaped fuzzy intervals. Inf Sci 177(18): 3831-3839

Hwang SY, Lee HS (2001) Nilpotent t-normbased sum of fuzzy intervals. Fuzzy Sets Syst 123(1): 73-80

Kar S, Majumder DD (2017) An investigative study on early diagnosis of prostate cancer using neuro-fuzzy classification system for pattern recognition. Int J Fuzzy Syst 19(2): 423439

Kaur J, Kumar A (2012) Exact fuzzy optimal solution of fully fuzzy linear programming problems with unrestricted fuzzy variables. Appl Intell 37(1): 145-154

Kaur J, Kumar A (2013) Mehar's method for solving fully fuzzy linear programming problems with L-R fuzzy parameters. Appl Math Model 37(12-13): 7142-7153

Ke H, Wu H, Huang H, Chen Z (2018) Optimal pricing decisions for a closed-loop supply chain with retail competition under fuzziness. J Oper Res Soc 69(9): 1468-1482

Li X (2015) A numerical-integration-based simulation algorithm for expected values of strictly monotone functions of ordinary fuzzy variables. IEEE Trans Fuzzy Syst 23(4): 964972

Liu B (1998) Minimax chance constrained programming models for fuzzy decision systems. Inf Sci 112(1-4):25-38

Liu B (2002) Theory and Practice of Uncertain Programming. Physica-Verlag, Berlin
Liu B (2004) Uncertainty Theory: An Introduction to its Axiomatic Foundations. SpringerVerlag, Berlin

Liu B (2010) Uncertainty Theory: A Branch of Mathematics for Modeling Human Uncertainty. Springer-Verlag, Berlin

Liu B, Iwamura K (1998a) Chance constrained programming with fuzzy parameters. Fuzzy Sets Syst 94(2): 227-237

Liu B, Iwamura K (1998b) A note on chance constrained programming with fuzzy coefficients. Fuzzy Sets Syst 100(1-3): 229-233

Liu B, Liu Y (2002) Expected value of fuzzy variable and fuzzy expected value models. IEEE Trans Fuzzy Syst 10(4): 445-450

Liu J, Chen Y, Zhou J, Yi X (2016) An exact expected value-based method to prioritize engineering characteristics in fuzzy quality function deployment. Int J Fuzzy Syst 8(4): 630-646

Liu Y, Liu J, Wang K, Zhang H (2016) A theoretical extension on the operational law for monotone functions of uncertain variables. Soft Comput 20(11): 4363-4376

Liu Y, Miao Y, Pantelous AA, Zhou J, and Ji P (2020) On fuzzy simulations for expected values of functions of fuzzy numbers and intervals. IEEE Trans Fuzzy Syst DOI: 10.1109/TFUZZ.2020.2979112

Mak Z (2012) Real vector space of LR-fuzzy intervals with respect to the shape-preserving t-norm-based addition. Fuzzy Sets Syst 200: 136-149

Markova A (1997) T-sum of LR fuzzy numbers. Fuzzy Sets Syst 85(3): 379-384

Muzzioli S, De Baets B (2017) Fuzzy approaches to option price modeling. IEEE Trans Fuzzy Syst 25(2): 392-401

Oussalah M, De Schutter J (2003) Approximated fuzzy LR computation. Inf Sci 153: 155175

Shakeel M, Abdullah S, Shahzad M, Amin F, Mahmood T, Amin N (2019a) Pythagorean trapezoidal fuzzy geometric aggregation operators based on Einstein operations and their application in group decision making. J Intell Fuzzy Syst 36(1): 309-324

Shakeel M, Abdullah S, Shahzad M, Siddiqui N (2019b) Geometric aggregation operators with interval-valued Pythagorean trapezoidal fuzzy numbers based on Einstein operations and their application in group decision making. Int J Mach Learn Cybern 10(10): 2867- 
2886

Soltanpour A, Baroughi F, Alizadeh B (2019) Intuitionistic fuzzy inverse 1-median location problem on tree networks with value at risk objective. Soft Comput 23(17): 7843-7852

Stefanini L, Guerra ML (2017) On possibilistic representations of fuzzy intervals. Inf Sci 405: $33-54$

Wang R, Zhou J, Yi X, Pantelous AA (2018) Solving the green-fuzzy vehicle routing problem using a revised hybrid intelligent algorithm. J Ambient Intell Humaniz Comput 10(1): 321-332

Wang Y, Zhao M, Han Y, Zhou J (2017) A fuzzy expression way for air quality index with more comprehensive information. Sustainability 9(1): 83

Yang Y, Zhou J, Wang K, Pantelous AA (2019) A new solution approach to two-stage fuzzy location problems with risk control. Comput Ind Eng 131: 157-171

Zadeh LA (1965) Fuzzy sets. Inf Control 8(3): 338-353

Zadeh LA (1975) The concept of a linguistic variable and its application to approximate reasoning-I. Inf Sci 8(3): 199-249

Zhou J, Yang F, Wang K (2016) Fuzzy arithmetic on LR fuzzy numbers with application$\mathrm{s}$ to fuzzy programming. J Intell Fuzzy Syst 30(1): 71-87

Zhong S, Pantelous AA, Goh M, Zhou J (2019) A reliability-and-cost-based fuzzy approach to optimize preventive maintenance scheduling for offshore wind farms. Mech Syst Signal Proc 124: 643-663

\section{Appendix A The abbreviations}

Table $\mathbf{5}$ The abbreviations are used in this manuscript

LR-FI: LR fuzzy interval
EV: expected value
ICD: inverse credibility distribution
CCP: chance-constrained programming
SDA: stochastic discretization algorithm
HIA: hybrid intelligent algorithm
NIA: numerical integration algorithm for LR-FIs
NIA-GA: an algorithm by integrating NIA with
genetic algorithm
$\mathcal{T}(\underline{c}, \bar{c}, \rho, \sigma)_{L R}:$ trapezoidal fuzzy number
$\mathcal{A}(\underline{c}, \bar{c}, \rho, \sigma)_{L R}, \mathcal{B}(\underline{c}, \bar{c}, \rho, \sigma)_{L R}:$ two specified
regular LR-FIs

\section{Appendix B The proof of Theorem 1}

Proof: For the case of $\delta=0$, we have $\operatorname{Cr}\{\zeta \leq$ $\left.\psi^{-1}(0)\right\}=\operatorname{Cr}\{\zeta \leq \sup \{t \mid \psi(t)=0\}\}=0$, thus Eq. (11) holds.

For any $\delta \in(0,1)$ and $\delta \in D_{\psi}$, it is obvious that there should be at least one point $t_{0}$ makes $\psi\left(t_{0}\right)=\delta$ holds. Thus $\operatorname{Cr}\left\{\zeta \leq t_{0}\right\}=\delta$ holds for any $t_{0} \in\{t \mid \psi(t)=\delta\}$, that is, $\operatorname{Cr}\left\{\zeta \leq f_{\delta}\right\}=\delta$ holds for $f_{\delta} \in \psi^{-1}(\delta)$. It is not difficult to find that $\sup \left\{\gamma \mid \psi^{-1}(\gamma)=\psi^{-1}(\delta)\right\}=\delta$. Eq. (11) is proved.

If $\delta \in(0,1)$ and $\alpha \notin D_{\Psi}$, in the light of Eq. (12), we can get $\operatorname{Cr}\left\{\zeta \leq \psi^{-1}(\delta)\right\}=\operatorname{Cr}\{\zeta \leq$ $\inf \{t \mid \psi(t) \geq \delta\}\}$. Let $\operatorname{Cr}\{\zeta \leq \inf \{t \mid \psi(t) \geq \delta\}\}=$ $\bar{\delta}$. Then we have $\bar{\delta}=\sup \left\{\gamma \mid \psi^{-1}(\gamma)=\psi^{-1}(\delta)\right\}$. Eq. (11) holds.

If $\delta=1, \operatorname{Cr}\left\{\zeta \leq \psi^{-1}(1)\right\}=\operatorname{Cr}\{\zeta \leq \inf \{t \mid \psi(t)$ $=1\}\}=1$. Eq. (11) is proved.

In the light of Definition $3, \psi^{-1}(\delta)$ is the ICD of $\zeta$.

\section{Appendix C The proof of Theorem 4}

Proof: For simplicity, we just consider the situation of $n=2$. Assume that

$\zeta=f\left(\zeta_{1}, \zeta_{2}\right)$,

where $f\left(\zeta_{1}, \zeta_{2}\right)$ is increasing for $\zeta_{1}$ and decreasing for $\zeta_{2}$. In addition, suppose that

$$
\begin{array}{r}
f\left(t_{1}, t_{2}\right)=s_{0}, \forall\left(t_{1}, t_{2}\right) \in\left\{\left(t_{1}, t_{2}\right) \mid \underline{\delta} \leq R_{1}\left(\frac{t_{1}-\overline{c_{1}}}{\sigma_{1}}\right)\right. \\
\left.\leq \bar{\delta}, \underline{\delta} \leq L_{2}\left(\frac{c_{2}-t_{2}}{\rho_{2}}\right) \leq \bar{\delta}\right\}
\end{array}
$$


where $s_{0}$ is a constant, $0<\underline{\delta}<\bar{\delta}<1$.

According to Zadeh's extensive principal, we know that $\nu(s)=\sup \left\{\nu_{1}\left(t_{1}\right) \wedge \nu_{2}\left(t_{2}\right) \mid f\left(t_{1}, t_{2}\right)=\right.$ $s\}$. Since $f\left(\zeta_{1}, \zeta_{2}\right)$ is increasing for $\zeta_{1}$ and decreasing for $\zeta_{2}$, and $\zeta_{1}$ and $\zeta_{2}$ are regular LRFIs, in view of Definition 1 , it can be obtained that $\forall t_{1} \in\left\{t_{1} \mid L_{1}\left(\frac{c_{1}-t_{1}}{\rho_{1}}\right) \in(0,1)\right\}, t_{2} \in\left\{t_{2} \mid R_{2}(\right.$ $\left.\left.\frac{t_{2}-\overline{c_{2}}}{\sigma_{2}}\right) \in(0,1)\right\}$,

$\nu(s)_{f\left(t_{1}, t_{2}\right)=s}=L_{1}\left(\frac{c_{1}-t_{1}}{\rho_{1}}\right)=R_{2}\left(\frac{t_{2}-\overline{c_{2}}}{\sigma_{2}}\right)$,

and $\forall t_{1} \in\left\{t_{1} \mid R_{1}\left(\frac{t_{1}-\overline{c_{1}}}{\sigma_{1}}\right) \in(0, \underline{\delta}) \cup(\bar{\delta}, 1)\right\}, t_{2} \in$ $\left\{t_{2} \mid\right.$

$\left.L_{2}\left(\frac{c_{2}-t_{2}}{\rho_{2}}\right) \in(0, \underline{\delta}) \cup(\bar{\delta}, 1)\right\}$,

$\nu(s)_{f\left(t_{1}, t_{2}\right)=s}=R_{1}\left(\frac{t_{1}-\underline{c_{1}}}{\sigma_{1}}\right)=L_{2}\left(\frac{\overline{c_{2}}-t_{2}}{\rho_{2}}\right)$.

Besides, it is easily known that $\nu\left(s_{0}\right)_{f\left(t_{1}, t_{2}\right)=s_{0}}=$ $\bar{\delta}$ for $\left(t_{1}, t_{2}\right) \in\left\{\left(t_{1}, t_{2}\right) \mid \underline{\delta} \leq R_{1}\left(\frac{t_{1}-\overline{c_{1}}}{\sigma_{1}}\right) \leq \bar{\delta}, \underline{\delta} \leq\right.$ $\left.L_{2}\left(\frac{c_{2}-t_{2}}{\rho_{2}}\right) \leq \bar{\delta}\right\}$. Based on the above analysis and Definition 1 , we can get that $\zeta=f\left(\zeta_{1}, \zeta_{2}\right)$ is an LR-FI.

\section{Appendix D The proof of Theorem 5}

Proof: According to Theorem 4, it is easily known that $\zeta$ is an LR-FI. Now we verify that Eq. (22) holds. For simplicity, we just verify the situation of $k=1$ and $n=2$. Assume that

$\zeta=f\left(\zeta_{1}, \zeta_{2}\right)$

where $f$ is increasing for $\zeta_{1}$ and decreasing for $\zeta_{2}$. In addition, suppose that

$\psi^{-1}(\delta)=f\left(\psi_{1}^{-1}(\delta), \psi_{2}^{-1}(1-\delta)\right)$,

where $\psi_{1}^{-1}$ and $\psi_{2}^{-1}$ are the ICDs of $\zeta_{1}$ and $\zeta_{2}$, respectively. For each $\delta \in[0,1]$, it is defined that

$$
\begin{gathered}
\bar{\delta}=\sup \left\{\gamma \mid f\left(\psi_{1}^{-1}(\gamma), \psi_{2}^{-1}(1-\gamma)\right)\right. \\
\left.=f\left(\psi_{1}^{-1}(\delta), \psi_{2}^{-1}(1-\delta)\right)\right\},
\end{gathered}
$$

which means that

$f\left(\psi_{1}^{-1}(\delta), \psi_{2}^{-1}(1-\delta)\right)=f\left(\psi_{1}^{-1}(\bar{\delta}), \psi_{2}^{-1}(1-\bar{\delta})\right)$

We know that $\psi_{1}^{-1}(\delta)$ and $\psi_{2}^{-1}(1-\delta)$ are both intervals or both points, in which points can be considered as a special kind of intervals.
Therefore, we only prove the case of intervals. The case of points can be verified similarly.

In view of Eq. (44), it is obvious that both $\psi_{1}^{-1}(\bar{\delta})$ and $\psi_{2}^{-1}(1-\bar{\delta})$ are also intervals. Then we can attain that, for $\forall t_{1} \in \psi_{1}^{-1}(\bar{\delta})$ and $\forall t_{2} \in$ $\psi_{2}^{-1}(1-\bar{\delta})$,

$f\left(t_{1}, t_{2}\right) \in \psi^{-1}(\bar{\delta})=\psi^{-1}(\delta)$.

For one thing, considering that $f$ is increasing for $\zeta_{1}$ and decreasing for $\zeta_{2}$, it can be deduced that

$\zeta_{1} \leq t_{1}$ and $\zeta_{2} \geq t_{2} \Rightarrow f\left(\zeta_{1}, \zeta_{2}\right) \leq f\left(t_{1}, t_{2}\right)$,

which means that

$\left\{\zeta_{1} \leq t_{1}\right\} \cap\left\{\zeta_{2} \geq t_{2}\right\} \subseteq\left\{f\left(\zeta_{1}, \zeta_{2}\right) \leq f\left(t_{1}, t_{2}\right)\right\}$.

In accordance with the increase of the credibility measure $\mathrm{Cr}$, we can obtain

$\operatorname{Cr}\left\{\zeta \leq f\left(t_{1}, t_{2}\right)\right\} \geq \operatorname{Cr}\left\{\left\{\zeta_{1} \leq t_{1}\right\} \cap\left\{\zeta_{2} \geq t_{2}\right\}\right\}$.

Then it can be attained that

$$
\begin{aligned}
& \operatorname{Cr}\left\{\left\{\zeta_{1} \leq t_{1}\right\} \cap\left\{\zeta_{2} \geq t_{2}\right\}\right\} \\
& =\operatorname{Cr}\left\{\zeta_{1} \leq t_{1}\right\} \wedge \operatorname{Cr}\left\{\zeta_{2} \geq t_{2}\right\} \\
& =\bar{\delta} \wedge \bar{\delta}=\bar{\delta} .
\end{aligned}
$$

In accordance with Eqs. (48) and (49), we get

$\operatorname{Cr}\left\{\zeta \leq f\left(t_{1}, t_{2}\right)\right\} \geq \bar{\delta}, \quad \forall f\left(t_{1}, t_{2}\right) \in \psi^{-1}(\delta)$.

For another thing, since $f$ is increasing for $\zeta_{1}$ and decreasing for $\zeta_{2}$, it can be deduced that

$f\left(\zeta_{1}, \zeta_{2}\right) \leq f\left(t_{1}, t_{2}\right) \Rightarrow \zeta_{1} \leq t_{1}$ or $\zeta_{2} \geq t_{2}$

Following from Eq. (51), we can get

$\left\{f\left(\zeta_{1}, \zeta_{2}\right) \leq f\left(t_{1}, t_{2}\right)\right\} \subseteq\left\{\zeta_{1} \leq t_{1}\right\} \cup\left\{\zeta_{2} \geq t_{2}\right\}$.

In terms of the increase of the credibility measure $\mathrm{Cr}$, it can be attained that

$\operatorname{Cr}\left\{\zeta \leq f\left(t_{1}, t_{2}\right)\right\} \leq \operatorname{Cr}\left\{\left\{\zeta_{1} \leq t_{1}\right\} \cup\left\{\zeta_{2} \geq t_{2}\right\}\right\}$. 
Then it can be derived that

$\operatorname{Cr}\left\{\left\{\zeta_{1} \leq t_{1}\right\} \cup\left\{\zeta_{2} \geq t_{2}\right\}\right\}$

$=\operatorname{Cr}\left\{\zeta_{1} \leq t_{1}\right\} \vee \operatorname{Cr}\left\{\zeta_{2} \geq t_{2}\right\}$

$=\bar{\delta} \vee \bar{\delta}=\bar{\delta}$.

In view of Eqs. (53) and (54), we have

$\operatorname{Cr}\left\{\zeta \leq f\left(t_{1}, t_{2}\right)\right\} \leq \bar{\delta}, \quad \forall f\left(t_{1}, t_{2}\right) \in \psi^{-1}(\delta)$.

Finally, combing Eqs. (50) and (55), we can get

$\operatorname{Cr}\left\{\zeta \leq f_{\delta}\right\}=\bar{\delta}, \quad \forall f\left(t_{1}, t_{2}\right)=f_{\delta} \in \psi^{-1}(\delta)$,

where $\bar{\delta}=\sup \left\{\gamma \mid \psi^{-1}(\gamma)=\psi^{-1}(\delta)\right\}$ holds in accordance with Eq. (43). In terms of Definition 3 , it can be known that $\psi^{-1}(\delta)=f\left(\psi_{1}^{-1}(\delta), \psi_{2}^{-1}\right.$ $(1-\delta))$ is just the ICD of $\zeta=f\left(\zeta_{1}, \zeta_{2}\right)$.

\section{Appendix E The proof of Theorem 6}

Proof: Following from Definition 6, we can get that

$$
\begin{aligned}
E[\zeta] & =\int_{0}^{+\infty} \operatorname{Cr}\{\zeta \geq t\} \mathrm{d} t-\int_{-\infty}^{0} \operatorname{Cr}\{\zeta \leq t\} \mathrm{d} t \\
& =\int_{0}^{+\infty}(1-\psi(t)) \mathrm{d} t-\int_{-\infty}^{0} \psi(t) \mathrm{d} t \\
& =\int_{0}^{+\infty} t \mathrm{~d} \psi(t) .
\end{aligned}
$$

By taking $\delta$ to replace $\psi(t)$ and $\psi^{-1}(\delta)$ to replace $t$, then it can be derived that

$$
E[\zeta]=\int_{0}^{1} \psi^{-1}(\delta) \mathrm{d} \delta .
$$

\section{Appendix F The proof of Theorem 8}

Proof: In the light of Theorem 7, it is deduced that

$$
\begin{aligned}
E[f(\boldsymbol{t}, \boldsymbol{\zeta})]= & \int_{0}^{1} f\left(\boldsymbol{t}, \psi_{1}^{-1}(\delta), \cdots, \psi_{k}^{-1}(\delta), \psi_{k+1}^{-1}\right. \\
& \left.(1-\delta), \cdots, \psi_{n}^{-1}(1-\delta)\right) \mathrm{d} \delta .
\end{aligned}
$$

In view of Theorem 5 , the ICD of $h_{v}\left(\boldsymbol{t}, \zeta_{1}, \zeta_{2}, \cdots\right.$, $\left.\zeta_{n}\right)$ is derived as

$$
\begin{aligned}
& \phi_{v}^{-1}(\delta)=h_{v}\left(\boldsymbol{t}, \psi_{1}^{-1}(\delta), \cdots, \psi_{k_{v}}^{-1}(\delta), \psi_{k_{v}+1}^{-1}(1-\delta),\right. \\
& \left.\cdots, \psi_{n}^{-1}(1-\delta)\right) . \\
& \text { It is not hard to find that } \operatorname{Cr}\left\{h_{v}\left(\boldsymbol{t}, \zeta_{1}, \zeta_{2}, \cdots, \zeta_{n}\right)\right. \\
& \leq 0\} \geq \delta \text { holds if and only if } \phi_{v}^{-1}(\delta) \leq 0 \text {. Espe- } \\
& \text { cially, when } \phi_{v}^{-1}(0.5) \text { is not unique, } \operatorname{Cr}\left\{h _ { v } \left(\boldsymbol{t}, \zeta_{1}, \zeta_{2},\right.\right. \\
& \left.\left.\cdots, \zeta_{n}\right) \leq 0\right\} \geq 0.5 \text { holds if only and if inf } \phi_{v}^{-1}(0.5) \\
& \leq 0 .
\end{aligned}
$$




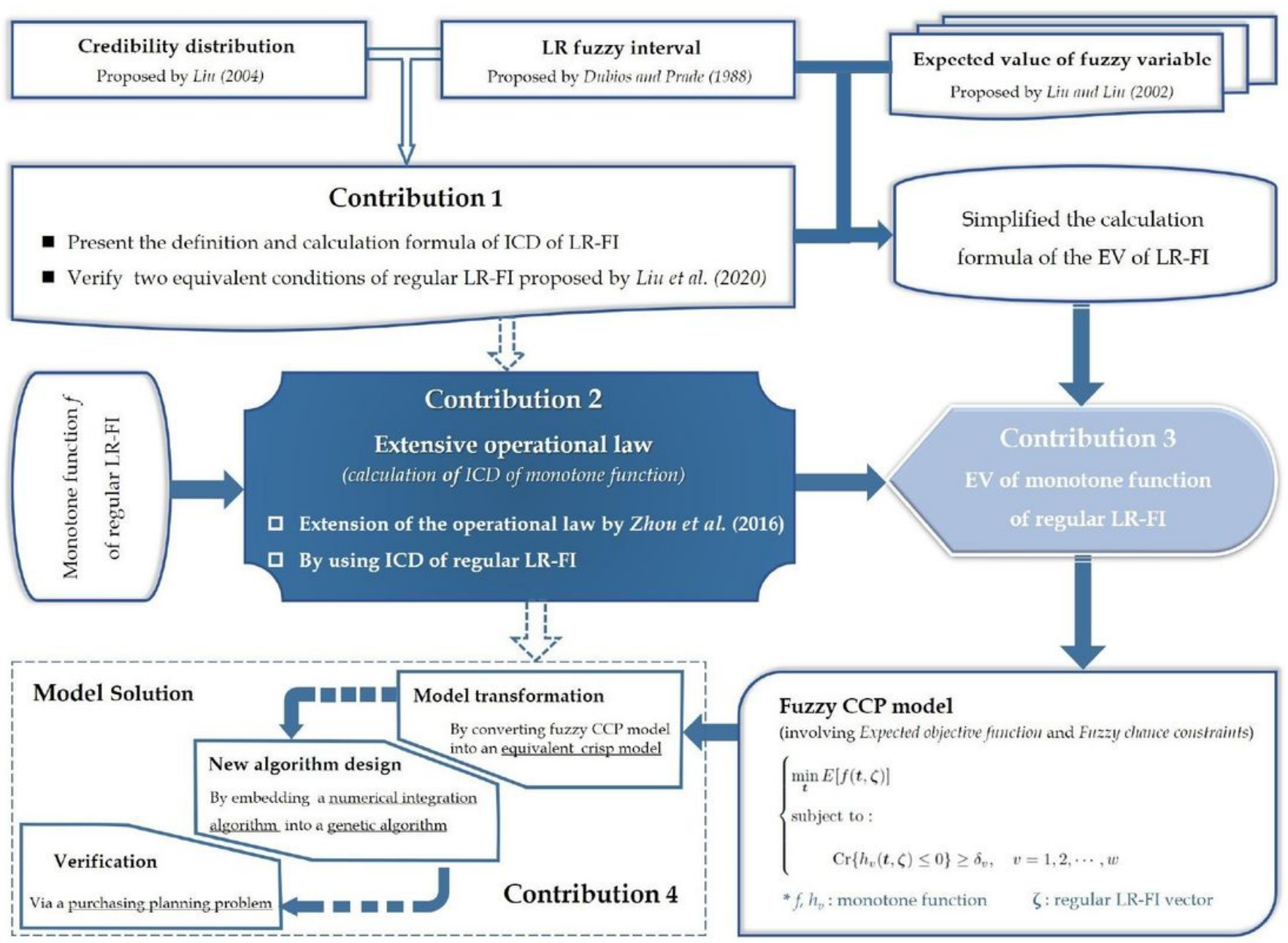

\section{Figure 1}

Please see the Manuscript PDF file for the complete figure caption
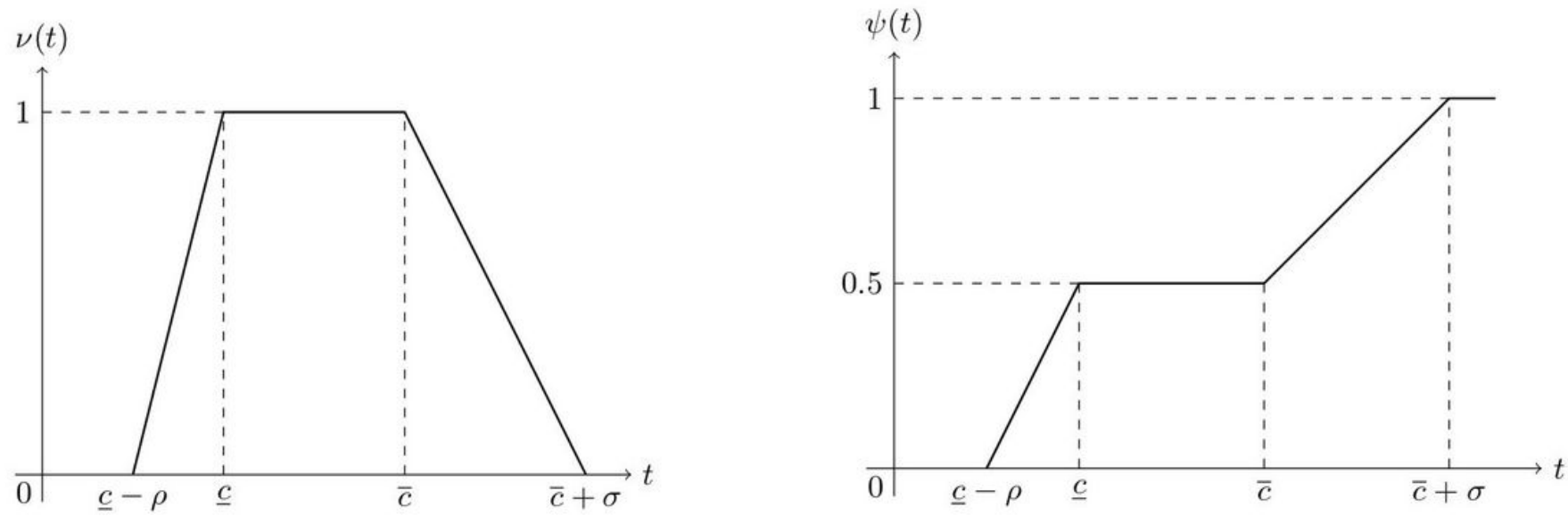

(a) The membership function

(b) The credibility distribution 
Figure 2

Please see the Manuscript PDF file for the complete figure caption

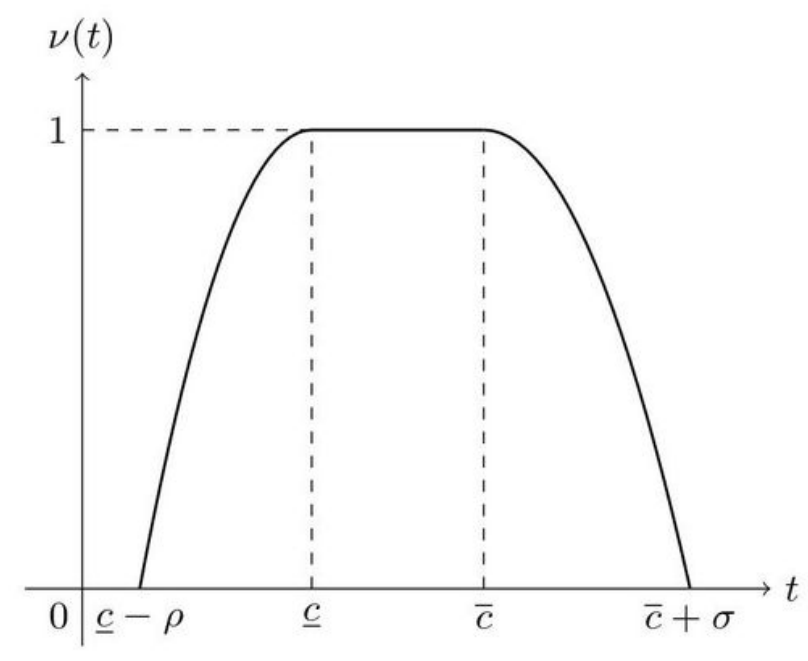

(a) The membership function

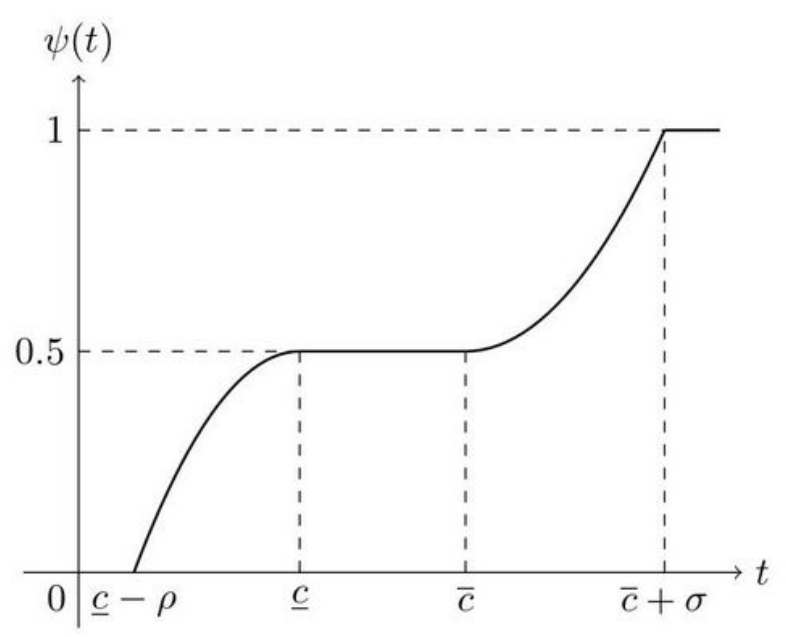

(b) The credibility distribution

\section{Figure 3}

Please see the Manuscript PDF file for the complete figure caption

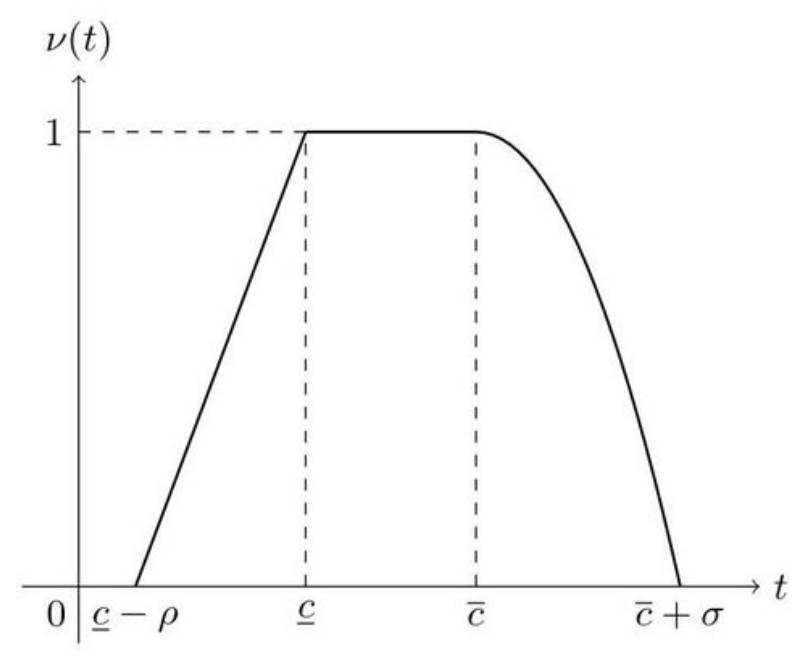

(a) The membership function

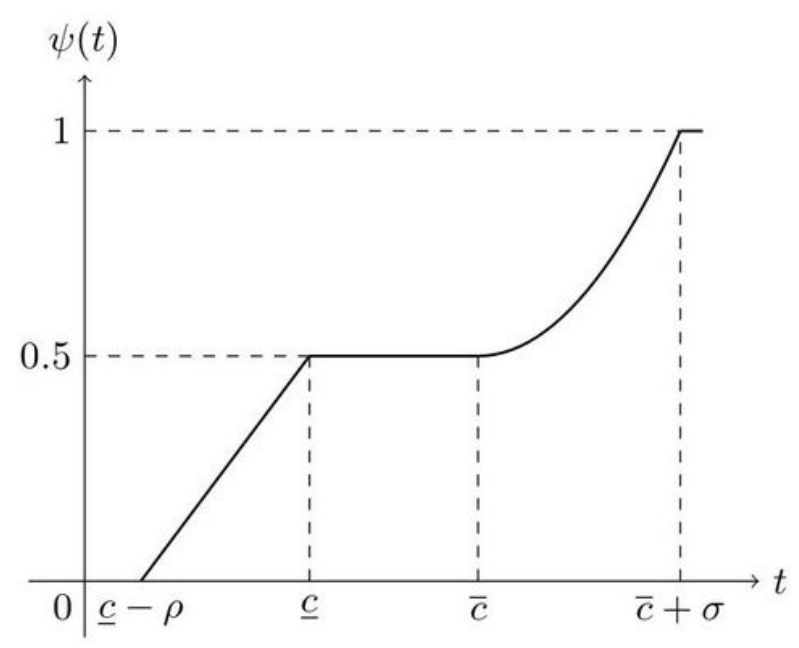

(b) The credibility distribution

\section{Figure 4}

Please see the Manuscript PDF file for the complete figure caption 


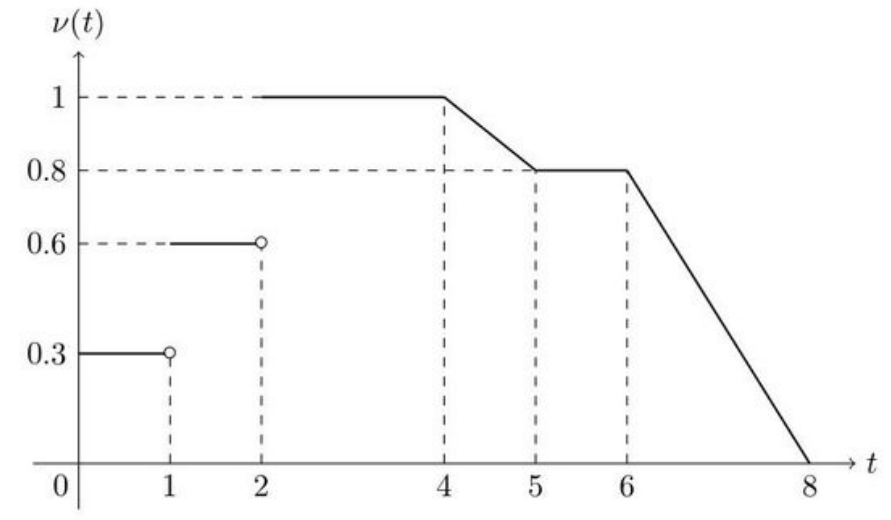

(a) The membership function

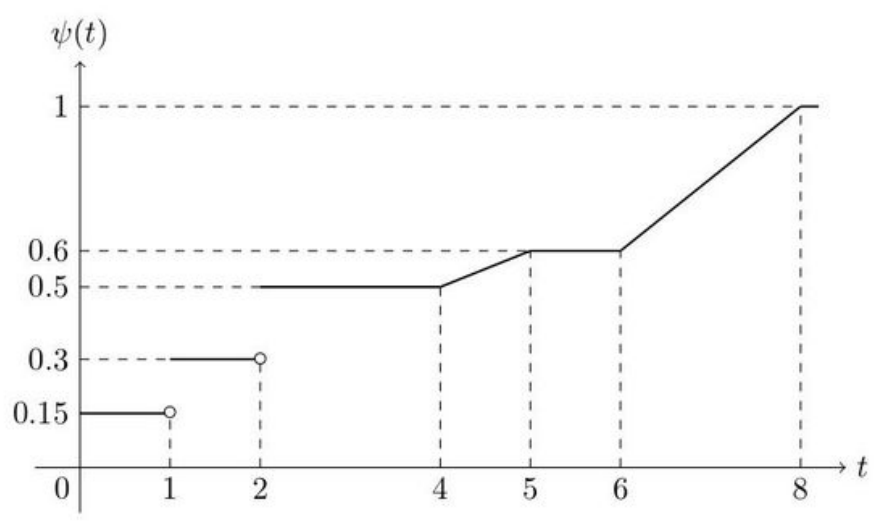

(b) The credibility distribution

\section{Figure 5}

Please see the Manuscript PDF file for the complete figure caption

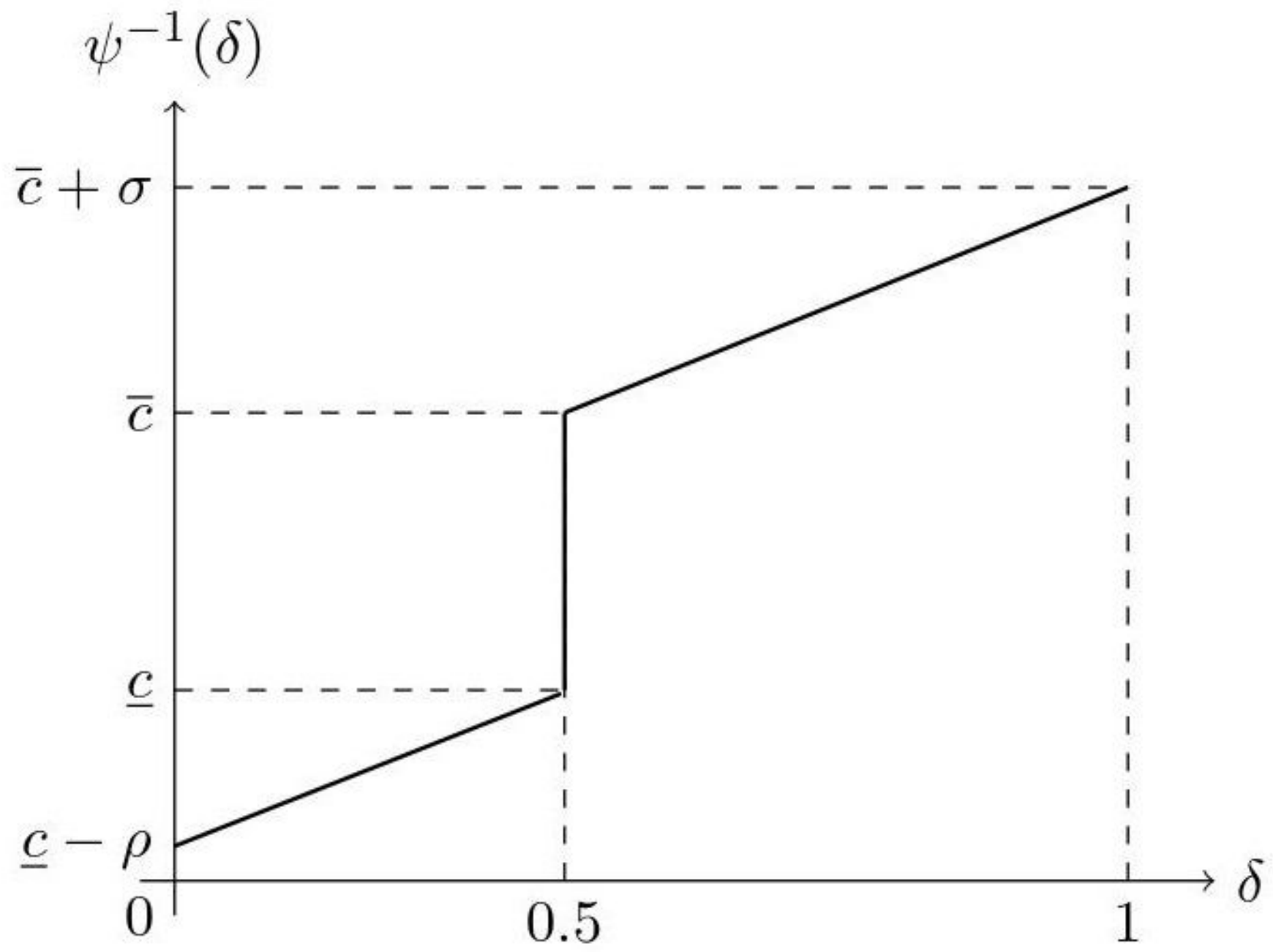


Figure 6

Please see the Manuscript PDF file for the complete figure caption

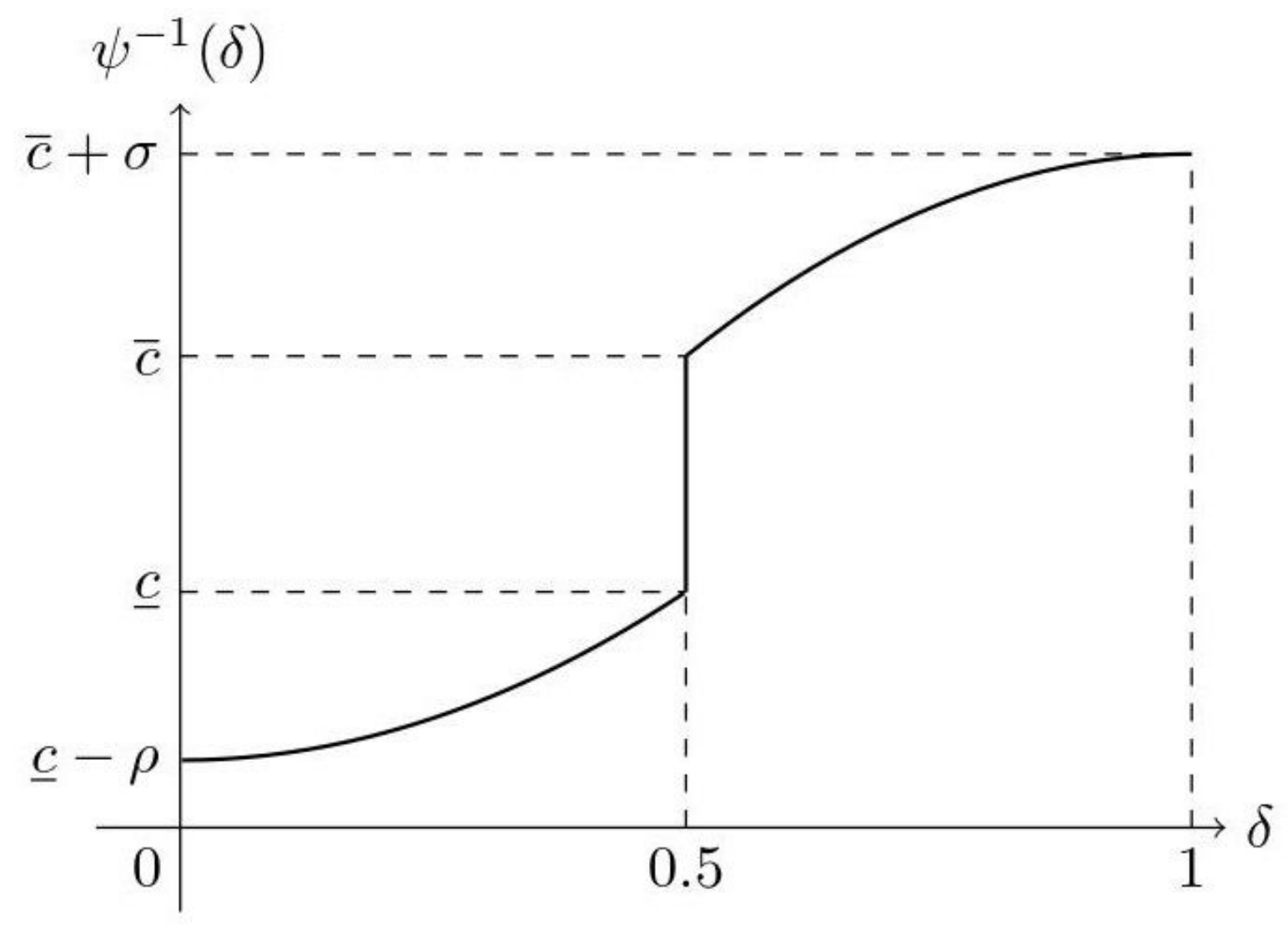

Figure 7

Please see the Manuscript PDF file for the complete figure caption 


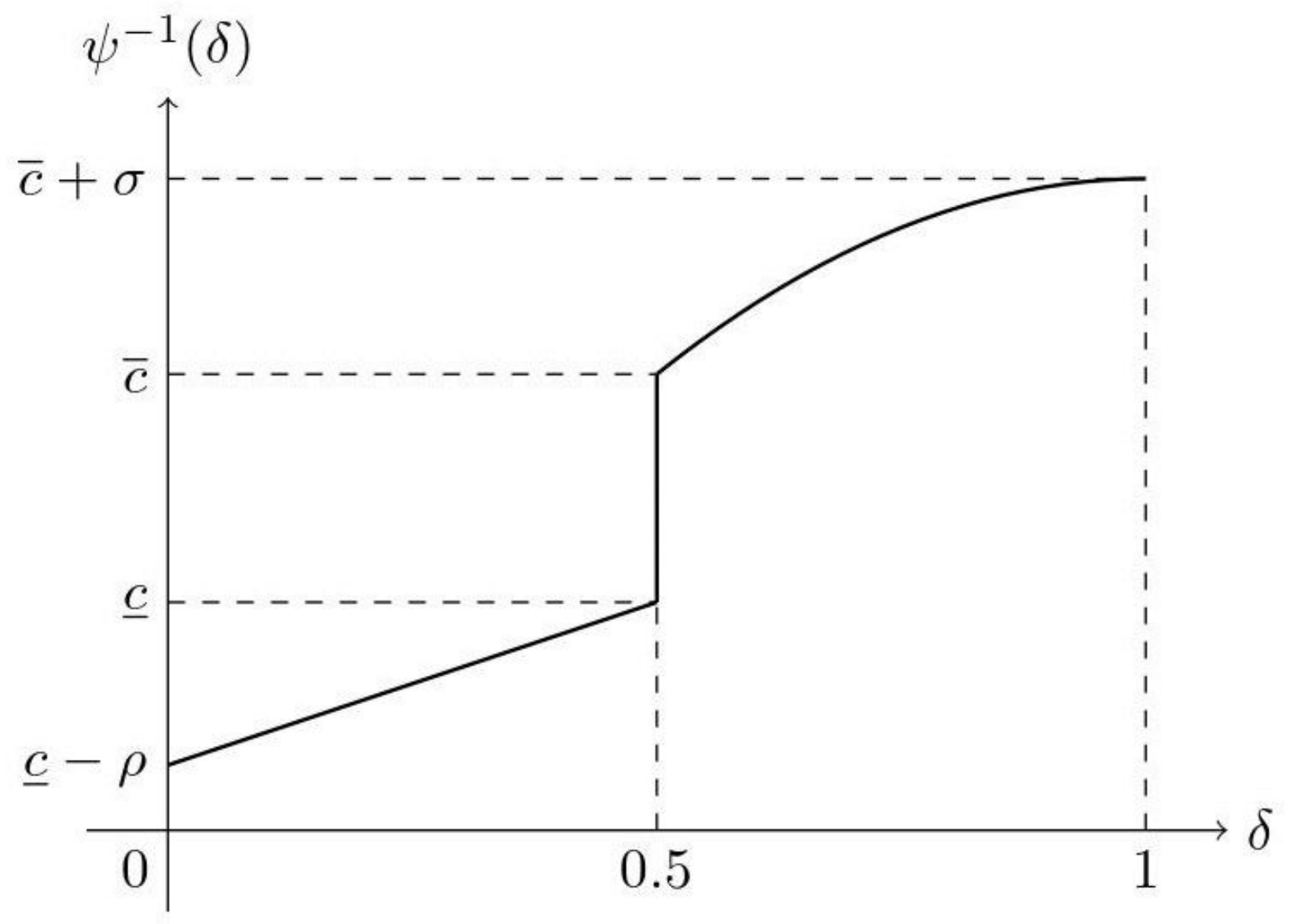

Figure 8

Please see the Manuscript PDF file for the complete figure caption 


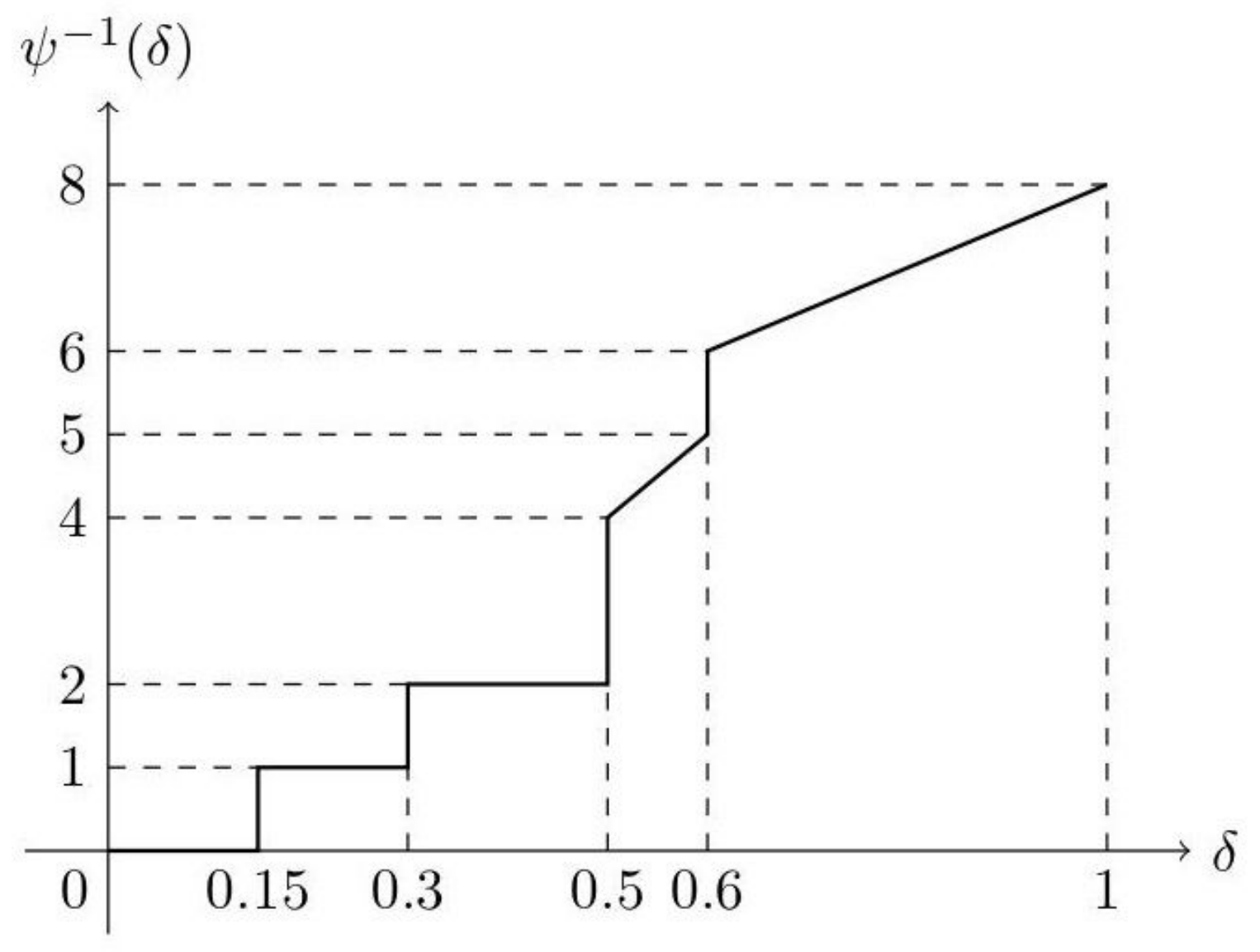

Figure 9

Please see the Manuscript PDF file for the complete figure caption 


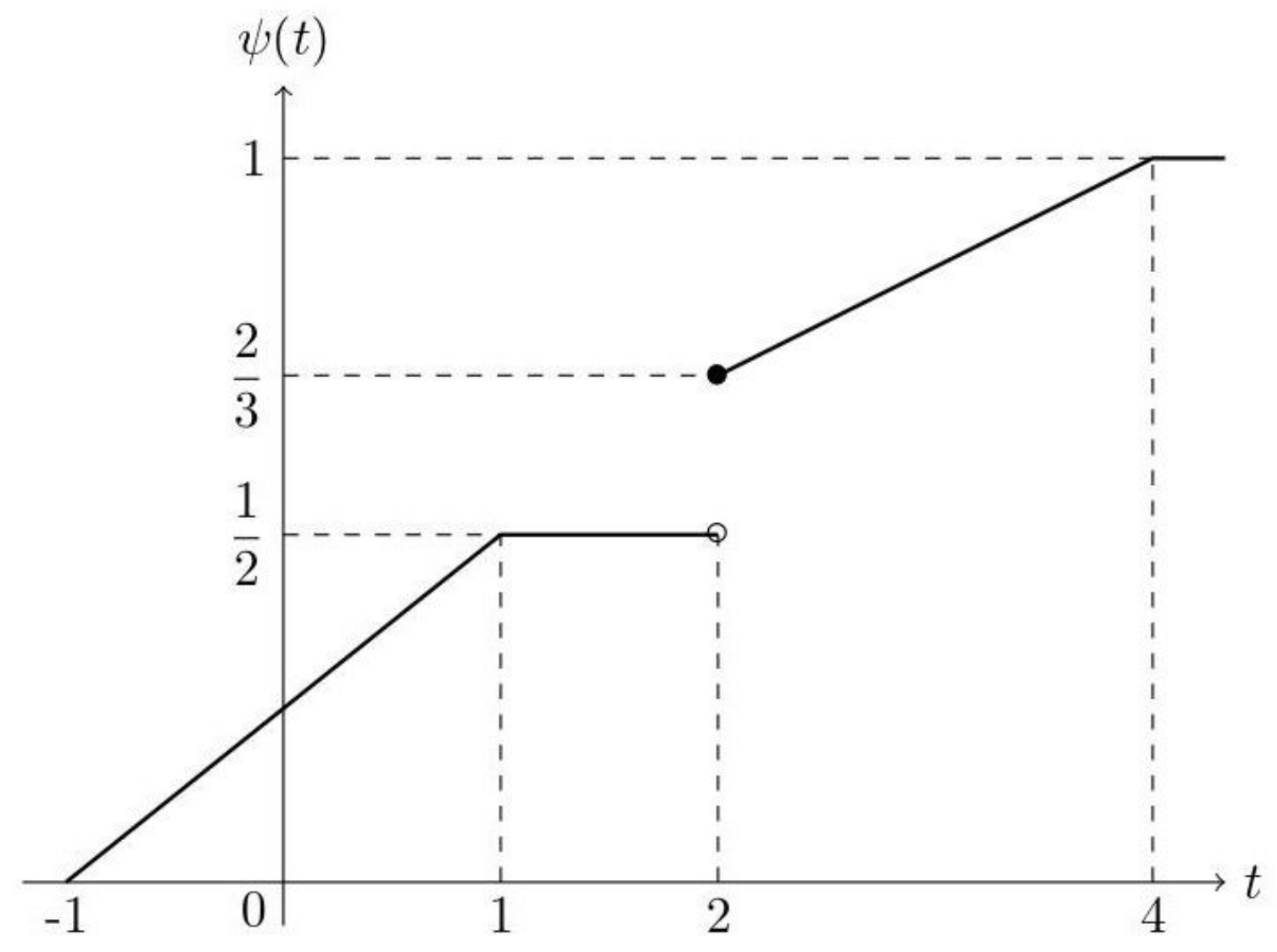

Figure 10

Please see the Manuscript PDF file for the complete figure caption 


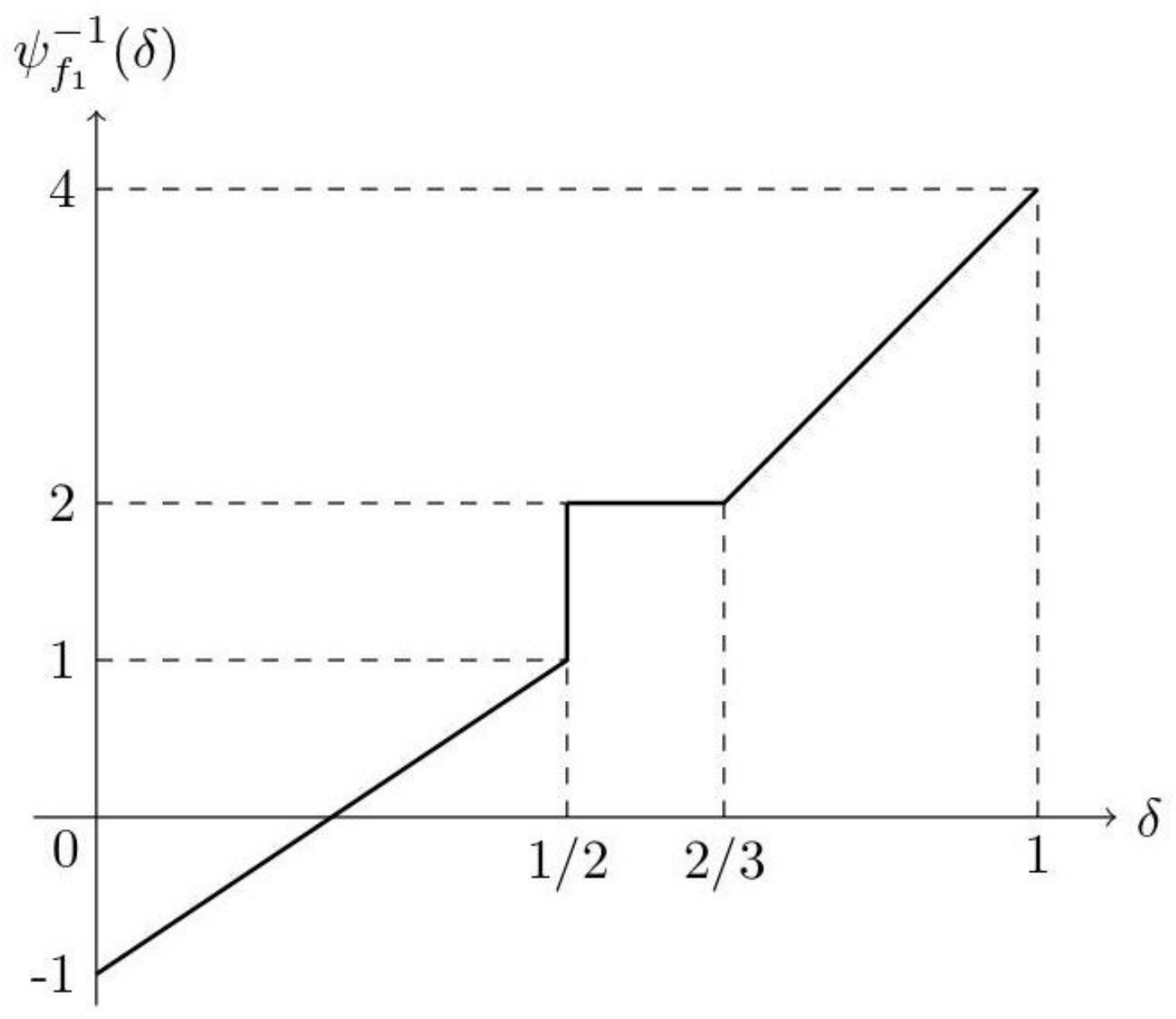

Figure 11

Please see the Manuscript PDF file for the complete figure caption 


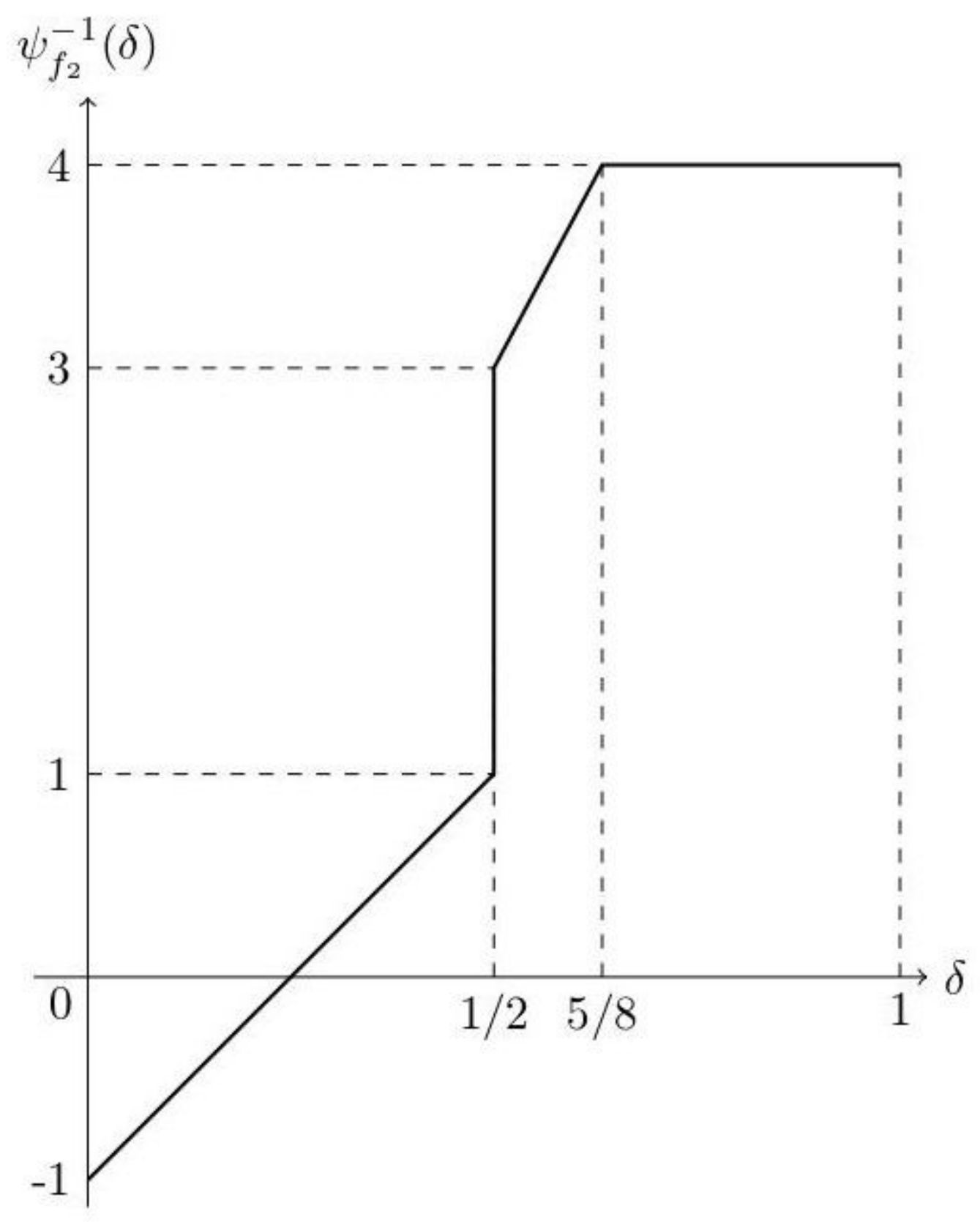

Figure 12

Please see the Manuscript PDF file for the complete figure caption 


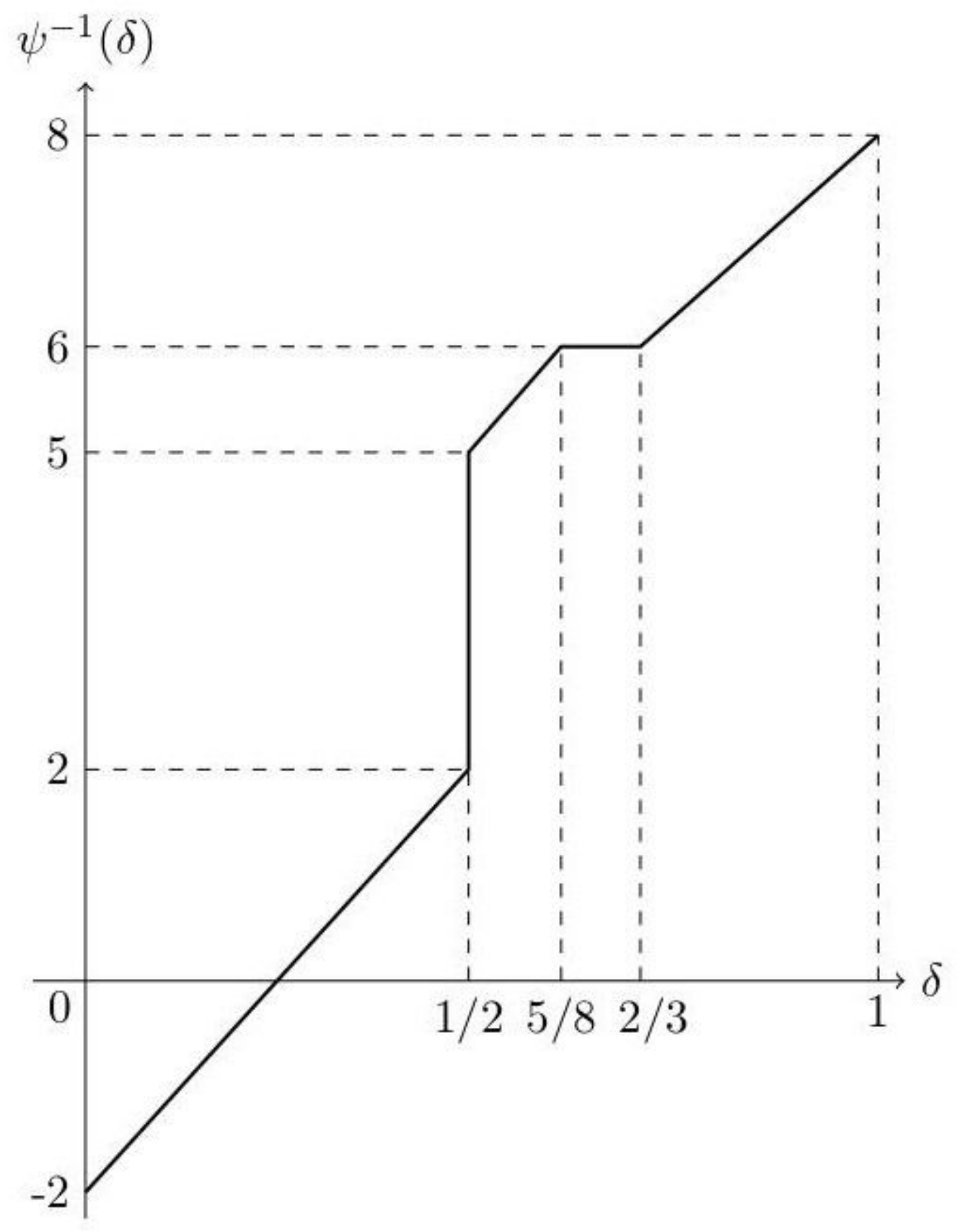

Figure 13

Please see the Manuscript PDF file for the complete figure caption 


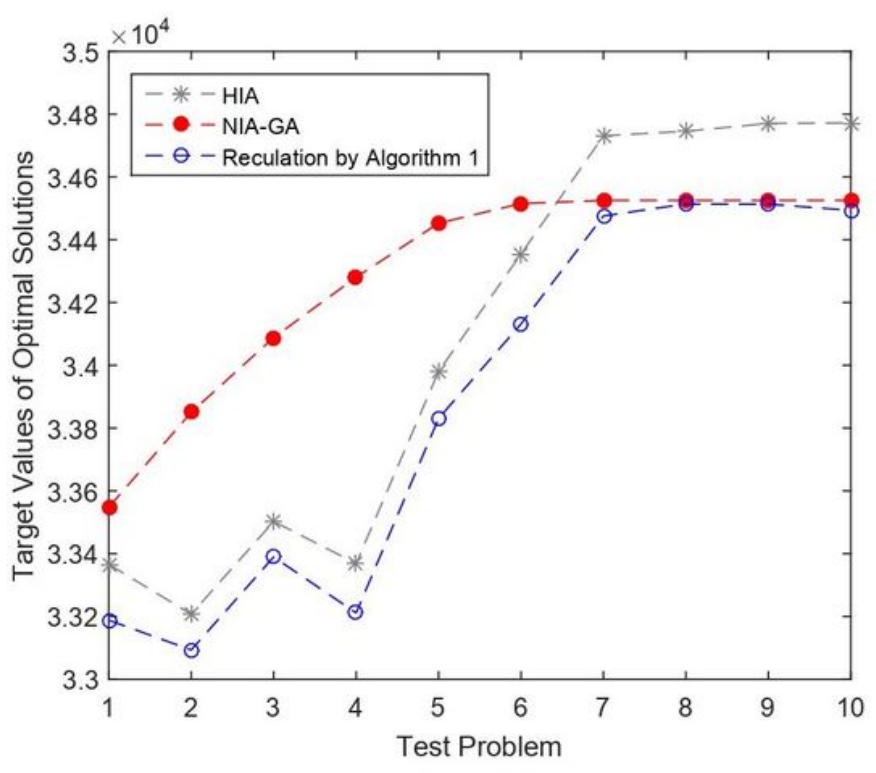

(a) $\delta_{0}=0.9$

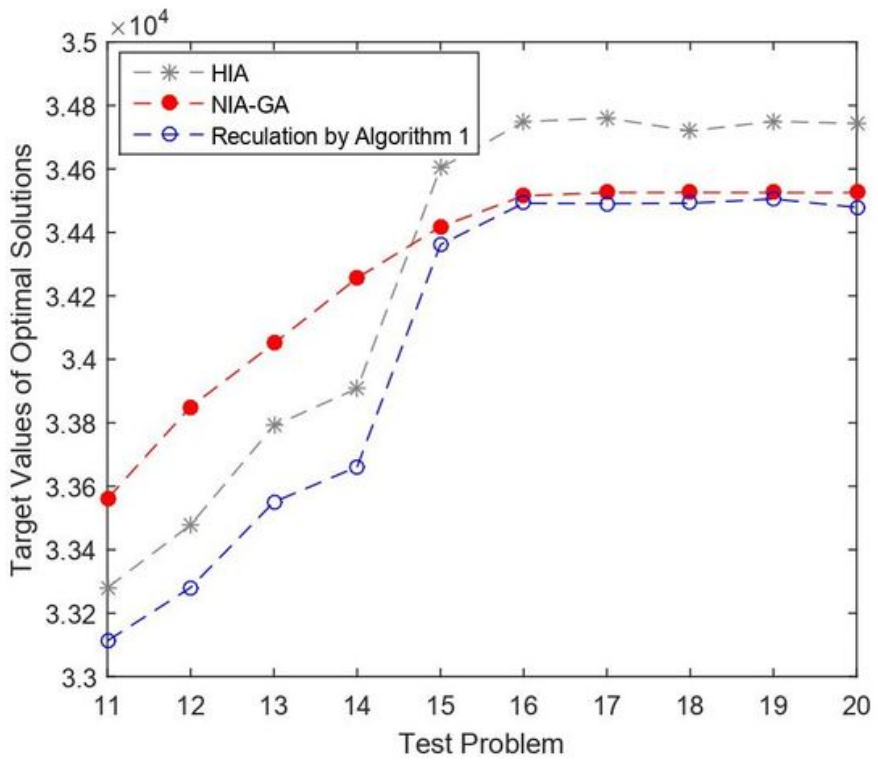

(b) $\delta_{0}=0.8$

Figure 14

Please see the Manuscript PDF file for the complete figure caption 